\title{
SKEW TENSOR PRODUCTS AND GROUPS OF CLASS TWO
}

\author{
PAUL CONRAD ${ }^{1)}$
}

\section{Introduction}

Let $\Delta$ and $N$ be abelian groups and let $f$ be a mapping of $\Delta \times \Delta$ into $N$ that is bilinear, skew symmetric and satisfies $f(\alpha, \alpha)=0$ for all $\alpha \in \Delta$. Such a mapping $f$ is called a (*)-mapping. By the Schreier extension theory $\Delta, N$ and $f$ determine a nilpotent group $G(\Delta, N, f)$ of class two that consists of the set $\Delta \times N$ with composition

$$
(\alpha, a)+(\beta, b)=(\alpha+\beta, f(\alpha, \beta)+a+b) .
$$

This paper is concerned with the class $\mathscr{S}$ of all nilpotent groups of class two that have a representation of the form $G(\Delta, N, f)$, where $f$ is a (*)-mapping and $\{f(\alpha, \beta): \alpha, \beta \in \Delta\}$ generates $N$. This class $\mathcal{S}$ is quite large. For example, in Theorem 5.2 we prove that if $G$ is a central extension of $N$ by $\Delta$, and if $\Delta$ is torsion free or if $N$ contains no elements of order 2 , then $G$ is a subgroup of a group in $\mathscr{S}$. Also every complete ( $\equiv$ divisible) nilpotent group of class two belongs to $\mathscr{S}$ (Theorem 3.1).

Consider $G(\Delta, N, f)$ in $\mathscr{S}$ and let $\Delta \otimes^{\prime} \Delta$ be the tensor product of $\Delta$ with itself. Let $D$ be the subgroup of $\Delta \otimes^{\prime} \Delta$ that is generated by the diagonal elements $\alpha \otimes^{\prime} \alpha \in \Delta^{\prime} \Delta$. The group $\Delta \otimes \Delta=\left(\Delta \otimes^{\prime} \Delta\right) / D$ is called the skew tensor product of $\Delta$ with itself, and the mapping

$$
e(\alpha, \beta)=\alpha \otimes \beta=\alpha \otimes^{\prime} \beta+D
$$

is a (*)-mapping of $\Delta \times \Delta$ into $\Delta \otimes \Delta$ that generates $\Delta \otimes \Delta$. Thus we have a group $G(\Delta, \Delta \otimes \Delta, e)$ and a unique homomorphism $\psi$ of $\Delta \otimes \Delta$ onto $N$ such that $f(\alpha, \beta)=e(\alpha, \beta) \psi$. In Theorem 5.1 we show that the mapping $(\alpha, a) \rightarrow(\alpha, a \psi)$ is a homomorphism of $G(\Delta, \Delta \otimes \Delta, e)$ onto $G(\Delta, N, f)$. Thus every group in $\mathscr{I}$ has a representation as a homomorphic image of a group of the form

Received April 18, 1962.

1) This research was supported by a grant from the National Science Foundation. 
$G(\Delta, \Delta \otimes \Delta, e)$. We use this representation to determine the structure of the groups in $\mathscr{S}$. For example, Theorem 5.3 and its corollaries determine conditions that are necessary and sufficient for two groups in $\mathscr{S}$ to be isomorphic. In Section 6 a study is made of the groups in $\mathscr{S}$ for which $\Delta$ is a rational vector space.

The author would like to thank Professors A. H. Clifford and L. Fuchs for their many useful suggestions. In particular Proposition 4.10 and Theorem 4.2 and their proofs are due to L. Fuchs.

\section{Representations and Homomorphisms of Nilpotent Groups of Class 2.}

Throughout this section let $G$ be a nilpotent group of class 2 with center $Z$ and commutator subgroup $C$, and let $N$ be a subgroup of $G$ between $Z$ and $C, G \supseteq Z \supseteq N \supseteq C$. Thus $G$ is a central extension of $N$ by the abelian group $\Delta=G / N$. We shall denote the elements of $\Delta$ by $\theta, \alpha, \beta, \ldots$ and those of $N$ by $O, a, b, \ldots$ Let $\pi$ be the natural homomorphism of $G$ onto $\Delta$ and let $r$ be a mapping of $\Delta$ into $G$ such that $r(\theta)=0$ and $r(\alpha) \pi=\alpha$ for all $\alpha$ in $\Delta$. Let

$$
f(\alpha, \beta)=-r(\alpha+\beta)+r(\alpha)+r(\beta)
$$

for $\alpha, \beta \in \Delta$, and for all $(\alpha, a)$ and $(\beta, b)$ in $\Delta \times N$ define that

$$
(\alpha, a)+(\beta, b)=(\alpha+\beta, f(\alpha, \beta)+a+b) .
$$

Then by the extension theory of Schreier, $\Delta \times N$ is a group and the mapping of $r(\alpha)+a$ upon $(\alpha, a)$ is an isomorphism of $G$ onto $\Delta \times N$. We shall denote this representation of $G$ by $G(\Delta, N, f)$. It is easy to show that $f$ is a mapping of $\Delta \times \Delta$ into $N$ that satisfies

$$
\begin{aligned}
& f(\alpha, \theta)=f(\theta, \beta)=0, \text { and } \\
& f(\alpha, \beta+\gamma)+f(\beta, \gamma)=f(\alpha+\beta, \gamma)+f(\alpha, \beta) .
\end{aligned}
$$

Conversely if $f$ is such a mapping and if we use (2) to define addition in $\Delta \times N$, then the result is a group. If $N$ is generated by the set $\{f(\alpha, \beta): \alpha, \beta \in \Delta\}$, then we say that $f$ generates $N$.

Two central extensions of $N$ by $\Delta, G(\Delta, N, f)$ and $G(\Delta, N, g)$ are equivalent if there exists an isomorphism $\sigma$ of the first onto the second such that for all $(\alpha, a)$ in $G(\Delta, N, f),(\alpha, a)_{\sigma}=(\alpha, t(\alpha)+a)$, where $t$ is a mapping of $\Delta$ into $N$. Thus the two groups are equivalent if and only if there exists a mapping $t$ of 
$\Delta$ into $N$ such that for all $\alpha$ and $\beta$ in $\Delta$

$$
g(\alpha, \beta)-f(\alpha, \beta)=-t(\alpha+\beta)+t(\alpha)+t(\beta) .
$$

The concept of equivalence frees the representation of $G$ from the particular choice of the representation mapping $r$.

A mapping $f$ of $\Delta \times \Delta$ into $N$ is a (*)-mapping if for all $\alpha, \beta, \gamma \in \Delta$

$$
\begin{aligned}
& f(\alpha, \beta+\gamma)=f(\alpha, \beta)+\dot{f}(\alpha, \gamma)\} \\
& f(\alpha+\beta, \gamma)=f(a, \gamma)+f(\beta, \gamma)\} \text { (bilinear) } \\
& f(\alpha, \beta)=-f(\beta, \alpha) \quad \text { (skew-symmetric) } \\
& f(\alpha, \alpha)=0 \text {. }
\end{aligned}
$$

It is easy to verify that any (*)-mapping satisfies (3) and (4), and hence determines a central extension of $N$ by $\Delta$.

Given $G \supseteq Z \supseteq N \supseteq C$ and $G^{\prime} \supseteq Z^{\prime} \supseteq N^{\prime} \supseteq C^{\prime}$ let $\Delta=G / N$ and $\Delta^{\prime}=G^{\prime} / N^{\prime}$. Then $G$ and $G^{\prime}$ have representations $G(\Delta, N, f)$ and $G^{\prime}\left(\Delta^{\prime}, N^{\prime}, f^{\prime}\right)$. Suppose that $\pi$ is a homomorphism of $G$ onto $G^{\prime}$, and that $(\theta \times N) \pi=\theta^{\prime} \times N^{\prime}$. Then for $(\alpha, a)$ in $G$ we have

$$
\begin{aligned}
(\alpha, a) \pi & =(\alpha, 0) \pi+(\theta, a) \pi=\left(\alpha \pi_{1}, \alpha \pi_{2}\right)+\left(\theta, a \pi_{3}\right) \\
& =\left(\alpha \pi_{1}, \alpha \pi_{2}+a \pi_{3}\right)
\end{aligned}
$$

where $\pi_{3}$ is a homomorphism of $N$ onto $N^{\prime}$.

$$
\begin{aligned}
{[(\alpha, a)+(\beta, b)] \pi=} & (\alpha+\beta, f(\alpha, \beta)+a+b) \pi \\
= & \left((\alpha+\beta) \pi_{1},(\alpha+\beta) \pi_{2}+\right. \\
& \left.f(\alpha, \beta) \pi_{3}+a \pi_{3}+b \pi_{3}\right) \\
(\alpha, a) \pi+(\beta, b) \pi= & \left(\alpha \pi_{1}, \alpha \pi_{2}+a \pi_{3}\right)+\left(\beta \pi_{1}, \beta \pi_{2}+b \pi_{3}\right) \\
= & \left(\alpha \pi_{1}+\beta \pi_{1}, f^{\prime}\left(\alpha \pi_{1}, \beta \pi_{1}\right)\right. \\
& \left.+\alpha \pi_{2}+\beta \pi_{2}+a \pi_{3}+b \pi_{3}\right) .
\end{aligned}
$$

Therefore $\pi_{1}$ is a homomorphism of $\Delta$ onto $\Delta^{\prime}$, and for all $\alpha$ and $\beta$ in $\Delta$

$$
(\alpha+\beta) \pi_{2}-\alpha \pi_{2}-\beta \pi_{2}=f^{\prime}\left(\alpha \pi_{1}, \beta \pi_{1}\right)-f(\alpha, \beta) \pi_{3} .
$$

It is easy to show that $\pi$ is an isomorphism if and only if $\pi_{1}$ and $\pi_{3}$ are isomorphisms. Conversely suppose that $\pi_{1}$ is a homomorphism of $\Delta$ onto $\Delta^{\prime}, \pi_{3}$ is a homomorphism of $N$ onto $N^{\prime}$, and $\pi_{2}$ is a mapping of $\Delta$ into $N^{\prime}$ that satisfies (6). If for $(\alpha, a)$ in $G$ we define that $(\alpha, a) \pi=\left(\alpha \pi_{1}, a \pi_{2}+a \pi_{3}\right)$, then $\pi$ is a homomorphism of $G$ onto $G^{\prime}$ and $(\theta \times N)_{\pi}=\theta^{\prime} \times N^{\prime}$. 
Note that (6) means that $f^{\prime}\left(\alpha \pi_{1}, \beta \pi_{1}\right)$ and $f(\alpha, \beta) \pi_{3}$ are equivalent factor mappings of $\Delta \times \Delta$ into $N^{\prime}$. Thus for an appropriate choice of $f^{\prime}, \pi_{2}=0$ will be achieved. However, no factor mapping that is equivalent to a $(*)$-mapping is again a (*)-mapping provided that $2 \Delta=\Delta$ or that $N$ contains no element of order 2 (Cf. Theorem 3.1). Now suppose that $f$ and $f^{\prime}$ are (*)-mappings. Then the left hand side of equation (6) is symmetric and the right hand side is skew-symmetric. Thus for all $\alpha$ and $\beta$ in $\Delta$

$$
0=2\left[f^{\prime}\left(\alpha \pi_{1}, \beta \pi_{1}\right)-f(\alpha, \beta) \pi_{3}\right]=f^{\prime}\left(\alpha \pi_{1}, 2 \beta \pi_{1}\right)-f(\alpha, 2 \beta) \pi_{3} .
$$

Therefore, if $2 \Delta=\Delta$ or if $N^{\prime}$ contains no elements of order 2 , then for all $\alpha$ and $\beta$ in $\Delta$

$$
f(\alpha, \beta) \pi_{3}=f^{\prime}\left(\alpha \pi_{1}, \beta \pi_{1}\right),
$$

and $\pi_{2}$ is a homomorphism of $\Delta$ into $N^{\prime}$. We summarize these results :

THEOREM 2.1. If $\pi$ is a homomorphism of $G(\Delta, N, f)$ onto $G^{\prime}\left(\Delta^{\prime}, N^{\prime}, f^{\prime}\right)$ such that $(\theta \times N) \pi=\theta^{\prime} \times N^{\prime}$, then for each $(\alpha, a)$ in $G$

$$
(\alpha, a) \pi=\left(\alpha \pi_{1}, \alpha \pi_{2}+a \pi_{3}\right)
$$

where $\pi_{1}$ is a homomorphism of $\Delta$ onto $\Delta^{\prime}, \pi_{3}$ is a homomorphism of $N$ onto $N^{\prime}$, and $\pi_{2}$ is a mapping of $\triangle$ into $N^{\prime}$ that satisfies (6), and conversely. Moreover, $\pi$ is an isomorphism if and only if $\pi_{1}$ and $\pi_{2}$ are isomorphisms. If $2 \Delta=\Delta$ or if $N^{\prime}$ contains no elements of order 2 , and if $f$ and $f^{\prime}$ are (*)-mappings, then $\pi_{2}$ is a homomorphism and (6) is equivalent to (7).

We next prove a few basic propositions about nilpotent groups of class 2 that are determined by $(*)$-mappings. Let $G=G(\Delta, N, f)$, where $f$ is a (*). mapping.

Proposition 2.1. $n(\alpha, a)=(n \alpha, n a)$ for all $(\alpha, a)$ in $G$ and all integers $n$. In particular $G$ is a complete group if and only if both $\Delta$ and $N$ are complete, and $G$ is torsion free if and only if both $\Delta$ and $N$ are torsion free.

Proof. Since $-(\alpha, a)=(-\alpha,-a)$ it suffices to consider only .positive integers. Using induction we have

$$
\begin{aligned}
(n+1)(\alpha, a) & =n(\alpha, a)+(\alpha, a)=(n \alpha, n a)+(\alpha, a) \\
& =((n+1) \alpha, f(n \alpha, \alpha)+(n+1) a)
\end{aligned}
$$




$$
\begin{aligned}
& =((n+1) \alpha, n f(\alpha, \alpha)+(n+1) a) \\
& =((n+1) \alpha,(n+1) a) .
\end{aligned}
$$

Proposition 2.2. If $f$ generates $N$ and $n$ is a positive integer, then the following are equivalent:
(a) $n \Delta=\Delta$.
(b) $n \Delta=\Delta$ and $n N=N$.
(c) $n G=G$.

In particular, $G$ is complete if and only if $\Delta$ is complete.

Proof. It follows from Proposition 2.1 that (b) and (c) are equivalent, and clearly $(b)$ implies $(a)$. Assume that $n \Delta=\Delta$ and consider $x \in N$.

$$
x=\sum f\left(\alpha_{i}, \beta_{i}\right)=\sum f\left(n \bar{\alpha}_{i}, \beta_{i}\right)=n \sum f\left(\bar{\alpha}_{i}, \beta_{i}\right) \in n N,
$$

where $\bar{\alpha}_{i}$ is an element in $\Delta$ such that $n \bar{\alpha}_{i}=\alpha_{i}$. Therefore $n N=N$.

Proposition 2.3. $n G$ is a fully invariant subgroup of $G$ for every positive integer $n$.

$$
\text { Proof. } \begin{aligned}
n(\alpha, a) & -n(\beta, b)=(n \alpha, n a)+(-n \beta,-n b) \\
= & (n(\alpha-\beta), f(n \alpha,-n \beta)+n(a-b)) \\
& =n(\alpha-\beta, f(\alpha,-n \beta)+a-b) \in n G .
\end{aligned}
$$

Proposition 2.4. The following are equivalent:

(a) $G$ is complete.

(b) Every non-zero homomorphic image of $G$ is infinite.

(c) G contains no maximal normal subgroups.

Proof. Clearly (a) implies (b) since every homomorphic image of a complete group is complete. Suppose that $G$ satisfies (b) and that $M$ is a maximal normal subgroup of $G$. Then $H=G / M$ is infinite and simple, and hence $H$ is non-abelian. But $H$ must be abelian because either $H=Z(H)$ or $0=Z(H) \supseteq C(H)$. Therefore (b) implies (c). Finally suppose that $G$ satisfies (c), but that $G$ is not complete. Then there exists a positive prime $p$ such that $p G \neq G$. By Proposition 2.3, $H=G / p G$ is a group, and every element in $H$ has order $p$. If $H$ is abelian, then it is a vector space over the integers modulo $p$, and hence it has a maximal subgroup. If $H$ is non-abelian, then 
$H / C(H)$ is a (non-trivial) vector space over the integers modulo $p$ and hence has a maximal subgroup. In either case we get a maximal normal subgroup of $G$, but this contradicts property (c).

For $(\alpha, a)$ and $(\beta, b)$ in $G$ we have

$$
\begin{aligned}
{[(\alpha, a),(\beta, b)]=} & -(\alpha, a)-(\beta, b)+(\alpha, a)+(\beta, b) \\
= & -((\beta, b)+(\alpha, a))+(\alpha+\beta, f(\alpha, \beta)+a+b) \\
= & -((\beta+\alpha, 0)+(\theta, f(\beta, \alpha)+b+a) \\
& +(\alpha+\beta, 0)+(\theta, f(\alpha, \beta)+a+b) \\
= & (\theta, 2 f(\alpha, \beta))
\end{aligned}
$$

and

$$
\begin{aligned}
-(\alpha, a)+(\beta, b)+(\alpha, a) & =[(\alpha, a),-(\beta, b)]+(\beta, b) \\
& =(\theta, 2 f(\alpha,-\beta))+(\beta, b) \\
& =(\beta, 2 f(\beta, \alpha)+b) .
\end{aligned}
$$

Thus $(\alpha, a)$ and $(\beta, b)$ commute if and only if $2 f(\alpha, \beta)=0$; and $C \subseteq 2 N^{\prime}$, where $N^{\prime}=\theta \times N$.

Proposition 2.5. If $\Delta$ or $N$ consists of elements of order 2 , then $G$ is abelian. If $\alpha \in \Delta$ is of order 2 , then $(\alpha, a)$ belongs to the center $Z$ of $G$ for all $a \in N$. Thus if $G / Z$ contains an element of order 2 and if $G(G / Z, Z, g)$ is $a$ representation of $G$, then $g$ is not a (*)-mapping.

Proposition 2.6. Suppose that $f$ generates $N$.

(i) If $N=2 N$, then $N^{\prime}=C$.

(ii) If $\Delta=2 \Delta$, then $N=2 N, G=2 G$ and $N^{\prime}=C$.

Proof. If $x \in N^{\prime}=2 N^{\prime}$, then $x=2 y$, where $y \in N^{\prime}$, and hence $x=2 y=$ $2\left(\theta, \sum \dot{f}\left(\alpha_{i}, \beta_{i}\right)\right)=\left(\theta, \sum 2 f\left(\alpha_{i}, \beta_{i}\right)\right) \in C$. Thus (i) is true and (ii) is an immediate consequence of Proposition 2.2.

Note that if $N$ is torsion and contains no elements of order 2 , then $N=2 N$. In fact in this case, $a \rightarrow 2 a$ is an automorphism of $N$.

Theorem 2.2. Suppose that $G=G(\Delta, N, f)$, where $f$ is a (*)-mapping that generates $N$, and suppose that $\Delta=2 \Delta$ or that $a \rightarrow 2 a$ is an automorphism of $N$. Then $\theta \times N$ is the commutator subgroup of $G$, and $\pi$ is an automorphism of $G$ if and only if for $(\alpha, a)$ in $G,(\alpha, a) \pi=\left(\alpha \pi_{1}, \alpha \pi_{2}+a \pi_{3}\right)$, where $\pi_{1}$ is an automorphism of $\Delta, \pi_{3}$ is an automorphism of $N, \pi_{2}$ is a homomorphism of $\Delta$ into $N$, and for all $\alpha$ and $\beta$ in $\Delta, f(\alpha, \beta) \pi_{3}=f\left(\alpha \pi_{1}, \beta \pi_{1}\right)$. 
Proof. If $a \rightarrow 2 a$ is an automorphism of $N$, then $N=2 N$, and hence by Proposition 2.6, $N^{\prime}=C$. Also in this case $N^{\prime}$ contains no elements of order 2 . Thus this theorem is an immediate consequence of Theorem 2.1.

Every nilpotent group of class 2 determines a (*)-mapping. For let $H$ be such a group, and let $C$ be the commutator subgroup of $H$; for $C+a$ and $C+b$ in $\Delta=H / C$ define that

$$
h(C+a, C+b)=[a, b] .
$$

Then $h$ is a (*)-mapping of $\Delta \times \Delta$ into $C$ that generates $C$, and hence $h$ determines a central extension $\widetilde{H}$ of $C$ by $\Delta . \quad \widetilde{H}=\widetilde{H}(\Delta, C, h)$. We shall now consider the mapping $H \rightarrow \widetilde{H}$. The next proposition asserts that it is single valued but not one-one, and 2.8. says that there are many fixed points.

Proposition 2.7. If two nilpotent groups $H$ and $H^{\prime}$ of class 2 are isomorphic, then so are $\widetilde{H}$ and $\widetilde{H}^{\prime}$, but the converse is false.

Proof. Let $\sigma$ be an isomorphism of $H$ onto $H^{\prime}$ and define that

$$
(C+a, b) \tau=\left(C^{\prime}+a \sigma, b_{\sigma}\right)
$$

for all $(C+a, b)$ in $\widetilde{H}$. Then $\tau$ is an isomorphism of $\widetilde{H}$ onto $\widetilde{H}^{\prime}$. Suppose that $H$ is a non-abelian group of order 8 . Then $Z=C$ is of order 2 and $H / C$ is the four group. It follows from proposition 2.5 that $\widetilde{H}$ is abelian. Thus if $H$ and $H^{\prime}$ are the two non-abelian groups of order 8 , then $\widetilde{H}=\widetilde{H}^{\prime}$. Note that by proposition 2.5 there is no representation of $H$ or of $H^{\prime}$ where the factor function is a $(*)$-mapping.

Proposition 2.8. Let $G=G(\Delta, N, f)$, where $f$ is a skew-symmetric factor function that generates $N$. If $a \rightarrow 2 a$ is an automorphism of $N$, then $G$ and $G$ are isomorphic.

Proof. By Lemma 2.1 in [3] $f$ is bilinear and since $N$ contains no elements of order $2, f(\alpha, \alpha)=0$ for all $\alpha \in \Delta$. Thus $f$ is a (*)-mapping. By proposition 2.6, $N^{\prime}=C$. Let

$$
g(\alpha, \beta)=f(\alpha, \beta)-f(\beta, \alpha)=2 f(\alpha, \beta) .
$$

Let $\pi_{3}$ be the automorphism $a \rightarrow 2 a$ of $N$, let $\pi_{2}$ be the zero homomorphism of $\Delta$ into $C$, and let $\pi_{1}$ be the identity automorphism of $\Delta$. Then 


$$
g\left(\alpha \pi_{1}, \beta \pi_{1}\right)-f(\alpha, \beta) \pi_{3}=2 f(\alpha, \beta)-2 f(\alpha, \beta)=0,
$$

and hence by Theorem 2.1, the mapping $(\alpha, a) \rightarrow(\alpha, 2 a)$ is an isomorphism of $G$ onto $\widetilde{G}$.

Note that the Schreier extension theory yields nothing in this case. For if $G$ is equivalent to $\widetilde{G}$ where both are considered as central extensions of $C=C(G)$ by $\Delta=G / C$, then it is easy to show that $G$ is abelian. Also from proposition 2.8. and from Theorem 3.1 in the next section it follows that if $H$ is a complete nilpotent group of class 2, whose commutator subgroup contains no elements of order 2 , then $H \cong \widetilde{H}$.

Baer ([2], p. 290) proves that the following are equivalent:

(a) There exists a group $H$ such that $Z(H) \cong N$ and $H / Z(H) \cong \Delta$.

(b) There exists a (*)-mapping $f$ of $\Delta \times \Delta$ into $N$ such that if $f(\alpha, \beta)=0$ for all $\beta$ in $\Delta$, then $\alpha=\theta$.

TNEOREM 2.3. If $N=2 N$ or if $\Delta=2 \Delta$, then the following are equivalent:

(i) There exists a group $H$ such that $C(H) \cong N$ and $H / C(H) \cong d$.

(ii) There exists $a(*) \cdot$ mapping of $\Delta \times \Delta$ into $N$ that generates $N$.

Proof. If (i) is satisfied, then the commutator function

$$
g(C(H)+a, C(H)+b)=[a, b]
$$

and the given isomorphisms $\triangle \cong H / C(H)$ and $C(H) \cong N$ determine a (*). mapping $f$ of $\Delta \times \Delta$ into $N$ that generates $N$. Conversely if $f$ is such a mapping, then let $H=H(\Delta, N, f)$ and let $N^{\prime}=\theta \times N$. Then $H / N^{\prime} \cong \Delta$ and by proposition 2.6, $N^{\prime}=C(H)$.

Direct sums of central extensions with amalgamations. Let $G_{1}, \ldots, G_{n}$ be central extensions of $N$ by the abelian groups $\Delta_{1}, \ldots, \Delta_{n}$. Then each $G_{1}$ has a representation $G_{i}\left(\Delta_{i}, N, f_{i}\right)$. Let $S=\Delta_{1} \times \cdots \times \Delta_{n} \times N$ and define

$$
\begin{aligned}
& \left(\alpha_{1}, \ldots, \alpha_{n}, a\right)+\left(\beta_{1}, \ldots, \beta_{n}, b\right)= \\
& \left(\alpha_{1}+\beta_{1}, \ldots, \alpha_{n}+\beta_{n}, f_{1}\left(\alpha_{1}, \beta_{1}\right)+\cdots+f_{n}\left(\alpha_{n}, \beta_{n}\right)+a+b\right) .
\end{aligned}
$$

Then $S$ is a central extension of $N$ by the direct sum of the $\Delta_{i} . \quad S$ is called the direct sum of the central extensions $G_{i}$ of $N$ with amalgamated $N$. If the $f_{i}$ are all (*)-mappings, then so is $\sum f_{i}$, and if one of the $f_{i}$ generates $N$, then so does $\sum f_{i}$. 
Conversely, let $G$ be a central extension of $N$ by an abelian group and suppose that $G_{1}, \ldots, G_{n}$ are normal subgroups of $G$ such that

(a) each $G_{i}$ is a proper extension of $N$,

(b) $G=G_{1}+\cdots+G_{n}$, and

(c) $G_{i} \cap\left(G_{1}+\cdots+G_{i-1}+G_{i+1}+\cdots+G_{n}\right)=N$ for $i=1, \ldots, n$.

Then $G$ is isomorphic to a direct sum of the $G_{i}$ with amalgamated $N$.

\section{Complete Nilpotent Groups of Class 2}

Throughout this section assume that $G \supseteq Z \supseteq C$ and that $G$ is complete. Then by Theorem 4.1 in [3], $Z$ and $C$ are complete, and, of course, $\Delta=G / C$ is complete.

Theorem 3.1. Let $G(\Delta, C, h)$ be a representation of $G$. Then in the set of all factor mappings of $\Delta \times \Delta$ into $C$ that are equivalent to $h$ there is one and only one (*)-mapping, and this mapping generates $C$. The structure of an automorphism of $G$ is given by Theorem 2.2.

Proof. First assume that $f$ and $g$ are (*)-mappings of $\Delta \times \Delta$ into $C$ that determine equivalent representations of $G$. Then there exists a mapping $t$ of $\Delta$ into $C$ such that for all $\alpha$ and $\beta$ in $\Delta$

$$
g(\alpha, \beta)-f(\alpha, \beta)=-t(\alpha+\beta)+t(\alpha)+t(\beta) .
$$

The left hand side of this equation is skew-symmetric and the right hand side is symmetric. Thus $2(g(\alpha, \beta)-f(\alpha, \beta))=0$, and hence $g(\alpha, 2 \beta)=f(\alpha, 2 \beta)$ for all $\alpha$ and $\beta$ in $\Delta$. Therefore since $2 \Delta=\Delta, f=g$.

By Theorem 4.1 in [3], $G=G^{\prime} \oplus S$, where $Z=C \oplus S, G^{\prime} \supseteq C$ and $G^{\prime} / C$ is a rational vector space. Thus $A=G / C=G^{\prime} / C \oplus Z / C$. Let $r$ be a representation mapping of $G^{\prime} / C$ into $G$ and extend $r$ to $\Delta$ as follows: $r(C+s)=s$ for all $s$ in $S$ and $r(\alpha)=r\left(\alpha^{\prime}\right)+r\left(\alpha^{\prime \prime}\right)$, where $\alpha \in \Delta, \alpha^{\prime} \in G^{\prime} / C, \alpha^{\prime \prime} \in Z / C$ and $\alpha=\alpha^{\prime}+\alpha^{\prime \prime}$. Let $k$ be the factor function determined by $r$. Then

$$
k\left(\alpha^{\prime}+\alpha^{\prime \prime}, \beta^{\prime}+\beta^{\prime \prime}\right)=k\left(\alpha^{\prime}, \beta^{\prime}\right)=-r\left(\alpha^{\prime}+\beta^{\prime}\right)+r\left(\alpha^{\prime}\right)+r\left(\beta^{\prime}\right) .
$$

By Lemma 3.4 in [3] there exists a (*)-mapping $f^{\prime}$ of $G^{\prime} / C \times G^{\prime} / C$ into $C$ that is equivalent to $k$ on $G^{\prime} / C \times G^{\prime} / C$. For $\alpha$ and $\beta$ in $\Delta$ define that 


$$
f(\alpha, \beta)=f\left(\alpha^{\prime}+\alpha^{\prime \prime}, \beta^{\prime}+\beta^{\prime \prime}\right)=f^{\prime}\left(\alpha^{\prime}, \beta^{\prime}\right) .
$$

Then $f$ is a $(*)$-mapping that generates $C, f$ is equivalent to $k$ and $k$ is equivalent to $h$.

Since $C$ is complete, it is a direct summand of $Z, Z=C \oplus Q$. Let $G^{\prime}$ be a maximal subgroup of $G$ with center $C$. Then by Theorem 4.1 in [3], $G=G^{\prime} \oplus Q$. Thus $G^{\prime}$ is uniquely determined (to within an isomorphism) by $G$. $Q=T \oplus F$, where $T$ is the torsion subgroup of $Q$ and $F$ is a torsicn free subgroup of $Q$. Let $G^{\prime \prime}=G^{\prime} \oplus F$. Then $G^{\prime \prime}$ is complete, $C$ is the commutator subgroup of $G^{\prime \prime}$ and $G^{\prime \prime} / C \cong G^{\prime} / C \oplus F$ which is a rational vector space. Note that it follows that if $G$ is torsion, then $G$ is abelian.

Lemma 3.1. $G^{\prime \prime}$ is a maximal subgroup of $G$ such that $G^{\prime \prime} \supseteq C$ and $G^{\prime \prime} / C$ is torsion free, and every other such subgroup of $G$ is isomorphic to $G^{\prime \prime}$.

Proof. $G=G^{\prime} \oplus Q=G^{\prime} \oplus F \oplus T$. Thus it is clear that $G^{\prime \prime}$ is a maximal subgroup of $G$ such that $G^{\prime \prime} \supseteq C$ and $G^{\prime \prime} / C$ is torsion free. Now let $H$ be a maximal subgroup of $G$ such that $H \supseteq C$ and $H / C$ is torsion free. Let

$$
\bar{C}=\{g \in G: n g \in C \text { for some } n>0\} .
$$

Then $\bar{C}$ is a complete subgroup of $Z$ that contains $C$ (Lemma 3.2 in [3]). Thus $\bar{C}=C \oplus D$, and it suffices to show that $G=H \oplus D . \quad H / C \cap \bar{C} / C=0$ and since $\bar{C} / C$ is complete, there exists a subgroup $K$ of $G$ such that $K \supseteq H$ and $G / C=K / C \oplus \bar{C} / C . \quad D$ is normal in $G$ because $D \subseteq Z$ and $K$ is normal in $G$ because $K \supseteq C . \quad K \cap D=D \cap \bar{C} \cap D=C \cap D=0 . \quad K+D=K+C+D=K+\bar{C}=G$. Thus $G=K \oplus D$. If $C+k \in K / C$ and $C=n(C+k)$ for some $n>0$, then $n k \in C$ and hence $k \in \bar{C} \cap K=C$. Therefore $K / C$ is torsion free and by the maximality of $H, H=K$.

Thus while investigating the structure of complete nilpotent groups of class 2, we may restrict our attention to the following two classes:

$\mathscr{C}$ : All complete nilpotent groups of class 2 such that $Z(H)=C(H)$.

$\mathscr{D}:$ All complete nilpotent groups of class 2 such that $H / C(H)$ is torsion free.

If $G \in \mathscr{C}$, then by Theorem 4.1 in [3] $G / C$ is torsion free. Therefore $\mathscr{C} \subseteq \mathscr{D}$. If $G \in \mathscr{D}$, then by Theorem $3.1, G$ has a representation $G(\Delta, C, f)$, where 
$\Delta=G / C$ is a rational vector space and $f$ is a $(*)$-mapping that generates $C$. Moreover,

$$
Z=\{(\alpha, a) \in G: f(\alpha, \beta)=0 \text { for all } \beta \text { in } \Delta\} .
$$

Therefore $G \in \mathscr{C}$ if and only if $f(\alpha, \beta)=0$ for all $\beta$ in $\Delta$ implies that $\alpha=\theta$.

If $G$ is a complete torsion free nilpotent group of class 2 , then $G \in \mathscr{D}$. For $C$ is complete and by Lemma 3.2 in [3]

$$
\bar{C}=\{g \in G: n g \in C \text { for some } n>0\} \subseteq Z .
$$

It follows that $C=\bar{C}$ and hence $G / C$ is torsion free. Moreover, $G / Z$ is a rational vector space and $Z$ is complete. Thus there exists a representation $G(G / Z, Z, f)$ of $G$, where $f$ is a (*)-mapping. Let $N$ be the subgroup of $Z$ that is generated by $f$. Then $N$ is complete, and in fact, $N=C$. It follows that $G(G / Z, C, f)$ is a maximal subgroup of $G$ with center $\theta \times C$.

\section{Skew Tensor Products}

In this section we derive some properties of skew tensor products that will be used in the following sections. Many of the proofs will be omitted, since they can be proven by slight modifications of the proofs of the corresponding propositions for ordinary tensor products (see for example [4], Chapter XI).

Let $U$ and $V$ be abelian groups, and let $X$ be a free abelian group with $U \times V$ as a free set of generators. Let $Y$ be the subgroup of $X$ that is generated by all elements in $X$ of the following forms:

$$
\begin{aligned}
& (a, a) \text { all } a \in U \cap V, \\
& (a, b)+(b, a) \text { all } a, b \in U \cap V, \\
& (a, b+c)-(a, b)-(a, c) \text { all } a \in U \text { and } b, c \in V \text {, and } \\
& (a+b, c)-(a, c)-(b, c) \text { all } a, b \in U \text { and } c \in V .
\end{aligned}
$$

Let $G=X / Y$ and denote the coset that contains $(a, b)$ by $a \otimes b$. Then $G$ consists of all finite sums $\sum k_{i}\left(a_{i} \otimes b_{i}\right)$ subject to the relations

$$
\begin{aligned}
& a \otimes a=0 \text { all } a \in U \cap V, \\
& a \otimes b=-(b \otimes a) \text { all } a, b \in U \cap V, \\
& a \otimes(b+c)=a \otimes b+a \otimes c \text { all } a \in U \text { and } b, c \in V,
\end{aligned}
$$


and $(a+b) \otimes c=a \otimes c+b \otimes c$ all $a, b \in U$ and $c \in V . \quad G$ is said to be the skew tensor product of $U$ and $V$, and we shall denote it by $U \otimes V$.

Proposition 4.1. Let $U \otimes^{\prime} V$ be the ordinary tensor product of $U$ and $V$, and let $T$ be the subgroup of $U \otimes \prime V$ that is generated by

$$
\left\{a \otimes^{\prime} a, a \otimes^{\prime} b+b \otimes^{\prime} a: a, b \in U \cap V\right\} .
$$

Then the mapping of $\sum k_{i}\left(a_{i} \otimes b_{i}\right)$ upon $\sum k_{i}\left(a_{i} \otimes b_{i}\right)$ is a homomorphism of $U \otimes \prime V$ onto $U \otimes V$ with kernel $T$. In particular, if $U \cap V=0$ or if $U \cap V$ is the null set, then $U \otimes V=U \otimes \prime V$.

Note that if $U \cap V$ is a subsemigroup of both $U$ and $V$, then we can drop the condition that $(a, b)+(b, a) \in Y$ for all $a, b \in U \cap V$, for then $0=(a+b$, $a+b)=(a+b, a)+(a+b, b)=(a, a)+(b, a)+(a, b)+(b, b)=(b, a)+(a, b)$. Thus we have merely the ordinary tensor product with a suppressed diagonal.

It follows immediately from Proposition 4.1 that $U \otimes V \cong V \otimes U$, and for all $u \in U, v \in V$ and all integers $n$,

$$
n u \otimes v=n(u \otimes v)=u \otimes n v .
$$

In particular, $U \otimes V$ consists of all finite sums $\sum u_{i} \otimes v_{i}$ with $u_{i} \in U$ and $v_{i} \in V$.

Proposition 4.2. If $U$ is complete, then so is $U \otimes V$, and if $U$ is complete and $V$ is a torsion group, then $U \otimes V=0$.

A mapping $f$ of $U \times V$ into a group $H$ is a (*)-mapping if

$$
\begin{array}{ll}
f(a, b+c)=f(a, b)+f(a, c) & a \in U, \quad b, c \in V \\
f(a+b, c)=f(a, c)+f(b, c) & a, b \in U, \quad c \in V \\
f(a, b)=-f(b, a) & a, b \in U \cap V \\
f(a, a)=0 & a \in U \cap V .
\end{array}
$$

As before, we say that $f$ generates $H$ if $\{f(u, v): u \in U$ and $v \in V\}$ is a set of generators for $H$. For $(u, v) \in U \times V$ define that

$$
e(u, v)=u \otimes v .
$$

Proposition 4.3. The mapping $e$ is a (*)-mapping of $U \times V$ into $U \otimes V$ that generates $U \otimes V$. If $f$ is a (*)-mapping of $U \times V$ into an abelian group $H$, then there exists a unique homomorphism $\varphi$ of $U \otimes V$ into $H$ such that $f(u, v)$ $=e(u, v) \varphi$. If $f$ generates $H$, then $\varphi$ is a homomorphism of $U \otimes V$ onto $H$. 
Proposition 4.4. Let $\mathscr{H}$ be the set of all (*)-mappings of $U \times V$ into an abelian group $N$. For $f, g \in \mathscr{F}, u \in U$ and $v \in V$ define $(f+g)(u, v)=f(u, v)$ $+g(u, v)$. Then

$$
\mathscr{H} \cong \operatorname{Hom}(U \otimes V, N) \text {. }
$$

Proof. Consider $\varphi \in H=\operatorname{Hom}(U \otimes V, N)$, and define $f(u, v)=(u \otimes v) \varphi$ for all $u \in U$ and $v \in V$. It follows by a straight forward computation that $f \in \mathscr{F}$ and that the mapping $\sigma$ of $\varphi$ upon $f$ is an isomorphism of $H$ into $\mathscr{H}$. If $f \in \mathscr{F}$, then by Proposition 4.3 there exists $\varphi \in H$ such that $f(u, v) \equiv(u \otimes v) \varphi$. Therefore $\varphi$ is an isomorphism of $H$ onto $\mathscr{H}$.

Let $\mathscr{G}$ be the group of all non-equivalent central extensions of an abelian group $N$ by an abelian group 4 . By Theorem 2.1 in [3] and the remarks after it we obtain the following theorem:

THeOREM 4.1. If $a \rightarrow 2 a$ is an automorphism of $N$, and if $N$ is a direct summand of every abelian extension of $N$ by $A$, then

$$
\mathscr{G} \cong \mathscr{H} \cong \operatorname{Hom}(\Delta \otimes \Delta \rightarrow N) \text {. }
$$

In particular, if $N$ is divisible and contains no elements 2 , or if $\Delta$ is free and $a \rightarrow 2 a$ is an automorphism of $N$, then our conclusion follows.

If $U=[u]$ is a cyclic group with generater $u$, then

$$
\sum n_{i}\left(m_{i} u \otimes v_{i}\right)=\sum u \otimes n_{i} m_{i} v_{i}=u \otimes \sum n_{i} m_{i} v_{i} .
$$

Therefore $[u] \otimes V=\{u \otimes v: v \in V\}$.

Proposition 4.5. Suppose that $U=[u]$ is cyclic and that $[u] \cap V$ is a subgroup of both [u] and $V$. Let $k$ be the order of [u] if this order is finite and 0 otherwise. Then there exists a least non-negative integer $n$ such that $[u] \cap V=[n u]$, and the mapping of $u \otimes v$ upon $v+\left(k V+\left[n^{2} u\right]\right)$ is an isomorphism of $[u] \otimes V$ upon $V /\left(k V+\left[n^{2} u\right]\right)$.

Proof. Every element in $[u] \times V$ has a unique representation $(m u, v)$ where $0 \leq m<k$ if $k \neq 0$ and $m$ is unrestricted otherwise. Thus $(m u, v) \rightarrow m v$ is a (single valued) mapping of $[u] \times V$ onto $V$. Since $[u] \times V$ is a free set of generators for $X$, this mapping is induced by a unique homomorphism $\alpha$ of $X$ onto $V$. Let $\delta$ be the natural homomorphism of $X$ onto $X / Y=[u] \otimes V$, and let $\beta$ be the natural homomorphism of $V$ onto $V /\left(k V+\left[n^{2} u\right]\right)$. It follows by 
the usual induced homomorphism arguments that there exists an isomorphism $\varphi$ of $[u] \otimes V$ onto $V /\left(k V+\left[n^{2} u\right]\right)$ such that $\delta \varphi=\alpha \beta$. Thus $(u \otimes v) \varphi=(u, v) \delta \varphi$ $=(u, v) \alpha \beta=v \beta=v+\left(k V+\left[n^{2} u\right]\right)$.

Corollary I. If $U$ is a cyclic group, then $U \otimes U=0$.

COROLlaRY II. Suppose that $[u]$ is an infinite cyclic group, and that $[u] \cap V$ is a subgroup of both $[u]$ and $V$. Then $[u] \otimes V \cong V /\left[n^{2} u\right]$, and if $[u] \cap V=0$, then $[u] \otimes V \cong V$, and if $[u] \subseteq V$, then $[u] \otimes V \cong V /[u]$.

Corollary III. If [u] and [v] are finite cyclic groups of orders $k$ and $\ell$ respectively, and if $[u] \cap[v]=0$, then $[u] \otimes[v] \cong[v] /(k[v])$ which is a cyclic group of order $(k, \ell)$.

In order to avoid ambiguities, we shall adapt the following notation:

(1) $V=\sum_{r \in \Gamma} \boxplus V_{r} \quad$ internal direct sum

(2) $V=\sum_{r \in \Gamma} \oplus V_{r} \quad$ external direct sum.

Thus in (1) the $V_{r}$ are subgroups of $V$, while in (2) $V$ is the restricted Cartesian product of the groups $V_{r}$.

Proposition 4.6. Suppose that $V=\sum \pm V_{r}$, where the $r$ belong to a linearly ordered set $\Gamma$, and let $G_{\alpha \beta}$ be the subgroup of $V \otimes V$ that is generated by the set $\left\{v_{\alpha} \otimes v_{\beta}: v_{\alpha} \in V_{\alpha}\right.$ and $\left.v_{\beta} \in V_{\beta}\right\}$. Then $G_{\alpha \beta} \cong V_{\alpha} \otimes V_{\beta}$ and

$$
V \otimes V=\sum_{\alpha \leq \beta} \boxplus G_{\alpha \beta} \cong \sum_{\alpha \leq \beta} \oplus\left(V_{\alpha} \otimes V_{\beta}\right) \text {. }
$$

Proof. The natural (*)-mapping $e$ of $V \times V$ into $V \otimes V$ induces a (*)mapping of $V \times V$ into $G_{\alpha \beta}$ for each pair $\alpha, \beta$ in $\Gamma$. Thus by Proposition 4.3 there exists a unique homomorphism $\varphi_{\alpha \beta}$ of $V_{\alpha} \otimes V_{\beta}$ into $G_{\alpha \beta}$ such that $\left(v_{\alpha} \otimes v_{\beta}\right)$ $\varphi_{\alpha \beta}=e\left(v_{\alpha}, v_{\beta}\right)$. For $\left(\ldots, x_{\alpha \beta}, \ldots\right)$ in $W=\sum \oplus\left(V_{\alpha} \otimes V_{\beta}\right)$ define that

$\left(\ldots, x_{\alpha \beta}, \ldots\right) \varphi=\sum_{\alpha \leq \beta} x_{\alpha \beta} \varphi_{\alpha \beta}$. Then $\varphi$ is a homomorphism of $W$ into $V \otimes V$.

Consider $u=\sum u_{\alpha}$ and $v=\sum v_{\alpha}$ in $V$, and define that $f(u, v)=\left(\ldots, u_{\alpha} \otimes\right.$ $\left.\left.v_{\alpha}, \ldots: \ldots,\left(u_{\alpha} \otimes v_{\beta}-v_{\alpha} \otimes u_{\beta}\right), \ldots\right)\right) \alpha<\beta$. By a straightforward argument it follows that $f$ is a (*)-mapping of $V \times V$ into $W$. Thus by Proposition 4.3 there exists a homomorphism $\varphi^{\prime}$ of $V \otimes V$ into $W$ such that $f(u, v)$ $=e(u, v) \varphi^{\prime}$. It is easy to verify that $f(u, v)=f(u, v) \varphi \varphi^{\prime}$ and that $e(u, v)=$. 
$e(u, v) \varphi^{\prime} \varphi$ for all $u$ and $v$ in $V$. Thus since $e$ generates $V \otimes V$ and $f$ generates $W$, it follows that $\varphi$ and $\varphi^{\prime}$ are reciprocal isomorphisms.

Note that $e(u, v)=\sum e\left(u_{\alpha}, v_{\alpha}\right)+\sum_{\alpha<\beta} e\left(u_{\alpha}, v_{\beta}\right)-e\left(v_{\alpha}, u_{\beta}\right)$. We next consider some special cases of the above proposition.

Case I. $\mathrm{V}$ is a free abelian group. Let $\left\{v_{\Upsilon}: \gamma \in \Gamma\right\}$ be a free set of generators for $V$. By Proposition 4.5, $\left[v_{\alpha}\right] \otimes\left[v_{\beta}\right]$ is an infinite cyclic group if $\alpha \neq \beta$ and $\left[v_{\alpha}\right] \otimes\left[v_{\alpha}\right]=0$. Thus by Proposition 4.6

$$
V \otimes V=\sum_{\alpha<\beta} \oplus\left[v_{\alpha} \otimes v_{\beta}\right] \text {. }
$$

For elements $u=\sum x_{\curlyvee} v_{\curlyvee}$ and $v=\sum y_{\curlyvee} v_{\curlyvee}$ in $V$

$$
e(u, v)=\sum_{\alpha<\beta}\left(x_{\alpha} y_{\beta}-x_{\beta} y_{\alpha}\right)\left(v_{\alpha} \otimes v_{\beta}\right)
$$

Case $I I . \quad V$ is a finitely generated abelian group. Then

$$
V=T_{l} \boxplus \ldots T_{k} \boxplus I_{l} \boxplus \ldots \oplus I_{\ell}
$$

where $T_{i}$ are finite cyclic groups of order $t_{i}, t_{i}$ divides $t_{i+l}$, and the $I_{i}$ are infinite cyclic groups. It follows from Propositions 4.5 and 4.6 that

$$
\begin{aligned}
& V \otimes V=\text { free group of rank }\left(\ell^{2}-\ell\right) / 2 \\
& \quad \boxplus \text { a direct sum of } \ell \text { copies of } T_{i} \text { for } i=1, \ldots, k \\
& \boxplus \text { a direct sum of copies of } T_{i} \otimes T_{j} \text { for } 1 \leq i<j \leq \ell .
\end{aligned}
$$

Note that $T_{i} \otimes T_{j}(i<j)$ is a cyclic group of order $t_{i}$.

By using Propositions 4.5 and 4.6 we can also compute $V \otimes V$ when $V$ is a direct sum of cyclic groups.

Fuchs [4] has shown that if $V$ is torsion, then the ordinary tensor product $V \otimes^{\prime} V$ is a direct sum of cyclic groups. It follows from this result and from Proposition 4.1 that the skew tensor product $V \otimes V$ is also a direct sum of cyclic groups.

Case III. $V$ is a complete abelian group. Then $V=T \boxplus F$, where $T$ is the torsion subgroup of $V$ and $F$ is a torsion free subgroup of $V$. By Proposition 4. 6

$$
V \otimes V \cong(T \otimes T) \oplus(T \otimes F) \oplus(F \otimes F),
$$

and by Proposition 4.2, $T \otimes T=(T \otimes F)=0$. Therefore $V \otimes V \cong F \otimes F$, and 
without loss of generality we may assume that $V$ is a rational vector space. Let $\left\{v_{\gamma}: \gamma \in \Gamma\right\}$ be a basis for $V$. Then $V=\sum \boxplus R v_{r}$, where $R$ is the field of rational numbers and by Proposition 4.6

$$
V \otimes V \cong \sum_{\alpha<\beta} \oplus\left(R v_{\alpha} \otimes R v_{\beta}\right)
$$

If $m$ and $n>0$ are integers, $\alpha, \beta \in \Gamma$ and $x, y \in R$, then

$$
n\left((m / n) x v_{\alpha} \otimes y v_{\beta}\right)=m x v_{\alpha} \otimes y v_{\beta}=m\left(x v_{\alpha} \otimes y v_{\beta}\right) .
$$

Since by Proposition 4. 8, $V \otimes V$ is torsion free, it follows that

$$
(m / n)\left(x v_{\alpha} \otimes y v_{\beta}\right)=(m / n) x v_{\alpha} \otimes y v_{\beta}=x v_{\alpha} \otimes(m / n) y v_{\beta} .
$$

If $x \in R v_{\alpha} \otimes R v_{\alpha}$, then $x=\sum x_{i} v_{\alpha} \otimes y_{i} v_{\alpha}=\left(\sum x_{i} y_{i}\right)\left(v_{\alpha} \otimes v_{\alpha}\right)=0$, where the $x_{i}$ and the $y_{i}$ belong to $R$. Therefore $R v_{\alpha} \otimes R v_{\alpha}=0$.

Next we show that if $\alpha \neq \beta$, then $R v_{\alpha} \otimes R v_{\beta} \cong R$. For $x$ and $y$ in $R$ define that

$$
f\left(x v_{\alpha}, y v_{\beta}\right)=x y .
$$

Then since $R v_{\alpha} \cap R v_{\beta}=0$, it follows that $f$ is a (*)-mapping of $R v_{\alpha} \times R v_{\beta}$ into $R$. Therefore by Proposition 4.3 there exists a homomorphism $\varphi$ of $R v_{\alpha} \otimes R v_{\beta}$ onto $R$ such that $x y=f\left(x v_{\alpha}, y v_{\beta}\right)=\left(x v_{\alpha} \otimes y v_{\beta}\right) \varphi$. If $x \in R v_{\alpha} \otimes R v_{\beta}$ and $0=x \varphi$, then

$$
x=\sum x_{i} v_{\alpha} \otimes y_{i} v_{\beta}=\left(\sum x_{i} y_{i}\right)\left(v_{\alpha} \otimes v_{\beta}\right) .
$$

Thus $0=x \varphi=\sum x_{i} y_{i}$, and hence $x=0$. Therefore $\varphi$ is an isomorphism, and

$$
V \otimes V \cong \sum_{\alpha<\beta} \oplus\left(R v_{\alpha} \otimes R v_{\beta}\right)
$$

where each $R v_{\alpha} \otimes R v_{\beta}$ is a one dimensional rational vector space. Note that if $u=\sum x_{\alpha} v_{\alpha}$ and $v=\sum y_{\alpha} v_{\alpha}$ are elements in $V$, then

$$
e(u, v)=\sum_{\alpha<\beta}\left(x_{\alpha} y_{\beta}-x_{\beta} y_{\alpha}\right)\left(v_{\alpha} \otimes v_{\beta}\right) .
$$

In particular, $\left\{\boldsymbol{v}_{\alpha} \otimes v_{\beta} \mid \alpha<\beta\right\}$ is a basis for $V \otimes V$.

Once again let $U$ and $V$ be abelian groups and let $U_{1}$ and $V_{1}$ be subgroups of $U$ and $V$ respectively. It is easy to show that the mapping of $\sum x_{i} \otimes y_{i} \in U_{1}$ $\otimes V_{1}$ upon $\sum x_{i} \otimes y_{i} \in U \otimes V$ is a homomorphism-the natural homomorphism of $U_{1} \otimes V_{1}$ into $U \otimes V$. 
Proposition 4.7. Let $V_{1}$ be a subgroup of $V$. If $V_{1}$ is pure in $V$ or if $V$ is torsion free, then the natural homomorphism of $V_{1} \otimes V_{1}$ into $V \otimes V$ is an isomorphism.

Proposition 4.8. If $V$ is torsion free, then so is $V \otimes V$.

Proof. Suppose that $z=\sum x_{i} \otimes y_{i} \in V \otimes V$ and that $n z=0$ for some $n>0$. Let $V_{1}$ be the subgroup of $V$ generated by the $x_{i}$ and the $y_{i}$. Then $V_{1}$ is a free abelian group, and hence by Proposition 4.6 $V_{1} \otimes V_{1}$ is torsion free. But by Proposition 4.7 the natural homomorphism of $V_{1} \otimes V_{1}$ into $V \otimes V$ is an isomorphism. Thus it follows that $z=0$, and hence $V \otimes V$ is torsion free.

Proposition 4.9. If $\pi$ is an endomorphism of $V$, then the mapping $\pi^{\prime}$ of $\sum x_{i} \otimes y_{i}$ upon $\sum x_{i} \pi \otimes y_{i} \pi$ is an endomorphism of $V \otimes V$. If $\pi$ is an automorphism, then so is $\pi^{\prime}$. We say that $\pi^{\prime}$ is induced by $\pi$. The mapping $\pi \rightarrow \pi^{\prime}$ is a homomorphism with respect to multiplication. In particular, the induced automorphisms of $V \otimes V$ form a group.

Proposition 4.10. If $V=2 V$, then the mapping $x \rightarrow 2 x$ is an automorphism of $V \otimes V$.

Proof. Let $A$ be the set of all elements in $V$ whose order is a power of 2 . Then $A$ is a complete subgroup of $V$, and hence $V=A \oplus B$ where $B$ contains no elements of order 2 and $2 B=B$. Thus $x \rightarrow 2 x$ is an automorphism of $B$ and it follows from Proposition 4.9 that $y \rightarrow 2 y$ is an automorphism of $B \otimes B$.

$$
V \otimes V=(A \otimes A) \oplus(A \otimes B) \oplus(B \otimes B) .
$$

Since $A$ is divisible and torsion, $A \otimes A=0$ (Proposition 4.2). Consider $a \in A$ and $b \in B$. If $2^{n}$ is the order of $a$, then since $2^{n} B=B, a \otimes b=a \otimes 2^{n} c=2^{n} a \otimes c$ $=0 \otimes c=0$ for some $c \in B$. Thus $A \otimes B=0$ and hence $x \rightarrow 2 x$ is an automorphism of $V \otimes V=B \otimes B$.

CoRollary If $V=2 V$ and $V$ is a torsion group, and if $N$ is a subgroup of $V \otimes V$, then $x \rightarrow 2 x$ is an automorphism of $(V \otimes V) / N$.

Suppose that $V=2 V$ and let $V \otimes^{\prime} V$ be the ordinary tensor product of $V$ by itself. Then by the analogue of Proposition 4.10, the mapping $x \rightarrow 2 x$ is an automorphism of $V \otimes^{\prime} V$ and for all $a, b$ in $V$,

$$
a \otimes^{\prime} b=1 / 2\left(a \otimes^{\prime} b-b \otimes^{\prime} a\right)+1 / 2\left(a \otimes^{\prime} b+b \otimes^{\prime} a\right) .
$$


As in the theory of linear transformations, this is the unique splitting of $e^{\prime}(a, b)$ $=a \otimes ' b$ into its skew symmetric and symmetric parts.

$$
\text { Let } \begin{aligned}
S & =\left[a \otimes^{\prime} b-b \otimes^{\prime} a: a, b \in V\right] \\
T & =\left[a \otimes^{\prime} b+b \otimes^{\prime} a: a, b \in V\right] .
\end{aligned}
$$

Theorem 4.2. If $V=2 V$, then $V \otimes^{\prime} V=S \oplus T$ and $S$ is isomorphic to $V \otimes V$. Moreover, both $S$ and $T$ are characteristic subgroups of $V \otimes^{\prime} V$ with respect to the induced automorphisms of $V \otimes^{\prime} V$.

Proof. Clearly $V \otimes^{\prime} V=S \oplus T$. For $a, b \in V$, let

$$
f(a, b)=1 / 2\left(a \otimes^{\prime} b-b \otimes^{\prime} a\right) .
$$

Then $f$ is a $(*)$-mapping of $V \times V$ into $S$ that generates $S$. Thus by Proposition 4.3 there exists a unique homomorphism $\psi$ of $V \otimes V$ onto $S$ such that $(a \otimes b) \psi$ $=f(a, b)$. If $0=\left(\sum a_{i} \otimes b_{i}\right) \psi=\sum 1 / 2\left(a_{i} \otimes b_{i}-b_{i} \otimes \prime a_{i}\right)$, then

$$
\begin{aligned}
& \sum a_{i} \otimes b_{i}=\left(\sum a_{i} \otimes b_{i}\right) \pi= \\
& \quad\left(\sum 1 / 2\left(a_{i} \otimes^{\prime} b_{i}+b_{i} \otimes^{\prime} a_{i}\right)\right) \pi=0,
\end{aligned}
$$

where $\pi$ is the natural homomorphism of $V \otimes^{\prime} V$ onto $V \otimes V$ (see Proposition 4.1). Therefore $\psi$ is an isomorphism of $V \otimes V$ onto $S$.

We close this section by determining the structure of the induced automorphism group for $V \otimes V$, where $V$ is a rational vector space.

Let $\pi$ be an automorphism of $V$, let $\pi^{\prime}$ be the induced automorphism of $V \otimes V$, and let $v_{1}, v_{2}, \ldots$ be a basis for $V$. Then since $\pi$ is a linear transformation, for each $i=1,2, \ldots$,

$$
v_{i} \pi=a_{i 1} v_{1}+a_{i 2} v_{2}+a_{i 3} v_{3}+\cdots
$$

where all but a finite number of the rational numbers $a_{i j}$ are zero. Since $\left\{v_{i} \otimes v_{j}: i<j\right\}$ is a basis for $V \otimes V$, for $i<j$

$$
\begin{aligned}
v_{i} \pi \otimes & v_{j \pi}=\left(a_{i 1} v_{1}+a_{i 2} v_{2}+\cdots\right) \otimes\left(a_{j 1} v_{1}+a_{j 2} v_{2}+\cdots\right) \\
& =\left|\begin{array}{ll}
a_{i 1} & a_{i 2} \\
a_{j 1} & a_{j 2}
\end{array}\right|\left(v_{1} \otimes v_{2}\right)+\left|\begin{array}{ll}
a_{i 1} & a_{i 3} \\
a_{j 1} & a_{j 3}
\end{array}\right|\left(v_{1} \otimes v_{3}\right)+\cdots \\
& =\sum_{r<s}\left|\begin{array}{ll}
a_{i r} & a_{i s} \\
a_{j r} & a_{j s}
\end{array}\right|\left(v_{r} \otimes v_{s}\right) .
\end{aligned}
$$

In particular if $\operatorname{dim}(V)=n$ is finite, then $\operatorname{dim}(V \otimes V)=\left(n^{2}-n\right) / 2, \pi$ is determined by the non-singular rational matrix 


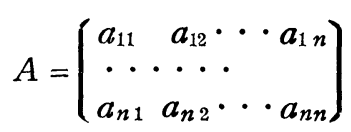

and $\pi^{\prime}$ is determined by the non-singular rational matrix

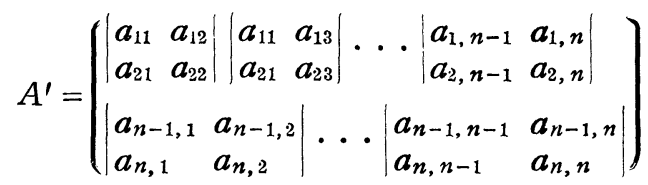

and the mapping $A \rightarrow A^{\prime}$ is a homomorphism.

Proposition 4.11. $\left|A^{\prime}\right|=|A|^{n-1}$.

Proof. $A=I_{1} \cdot I_{2} \cdots I_{k}$, where the $I_{i}$ are elementary matrices that are obtained from the $n \times n$ identity matrix $I$ by multiplying a row or column by $0 \neq d$ or by adding a row or column of $I$ to another row or column of $I$. Since the mapping $A \rightarrow A^{\prime}$ is a homomorphism, $A^{\prime}=I_{1}^{\prime} \cdot I_{2}^{\prime} \cdots I_{k}^{\prime}$. If $I_{i}$ is obtained by multiplying a row (or column) of $I$ by $0 \neq d$, then $\left|I_{i}\right|=d$ and $\left|I_{i}^{\prime}\right|=d^{n-1}$ because $I_{i}^{\prime}$ is the matrix obtained from the $\left(n^{2}-n\right) / 2 \times\left(n^{2}-n\right) / 2$ identity matrix $J$ by multiplying $n-1$ of the rows (or columns) of $J$ by $d$. If $I_{i}$ is obtained from $I$ by adding one row (or column) to another, then $\left|I_{i}\right|=\left|I_{i}^{\prime}\right|=1$. Therefore

$$
\left|A^{\prime}\right|=\left|I_{1}^{\prime}\right|\left|I_{2}^{\prime}\right| \cdots\left|I_{k}^{\prime}\right|=\left|I_{1}\right|^{n-1} \cdots\left|I_{k}\right|^{n-1}=|A|^{n-1} .
$$

Lemma 4.4. Let $A=\left(a_{i j}\right)$ be an $n \times n$ non-singular rational matrix, let $I$ be the $n \times n$ identity matrix, let $J$ be the $\left(n^{2}-n\right) / 2 \times\left(n^{2}-n\right) / 2$ identity matrix and let $A^{\prime}$ be as above. If $n \geq 3$ and $A^{\prime}=J$, then $A= \pm I$.

Proof. From

we have

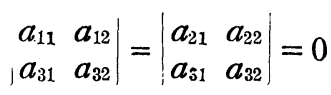

$$
\begin{aligned}
& a_{11} a_{32}+a_{12}\left(-a_{31}\right)=0 \\
& a_{21} a_{32}+a_{22}\left(-a_{31}\right)=0 .
\end{aligned}
$$

But because of

$$
\left|\begin{array}{ll}
a_{11} & a_{12} \\
a_{21} & a_{22}
\end{array}\right|=1 \neq 0
$$

we infer that $a_{32}=-a_{31}=0$. This argument shows (when $n \geq 3$ ) that $A$ must be a diagonal matrix. Then from $a_{11} a_{22}=a_{11} a_{33}=1$ etc., we get that $A$ must be 
a scaler matrix, and since $1=\left|A^{\prime}\right|=|A|^{n-1}, A= \pm I$.

THEOREM 4.3. Let $V$ be a rational vector space with (finite or infinite) dimension $\geq 3$. Tnen the kernel of the natural homomorphism of the group of automorphisms of $V$ onto the group of induced automorphisms of $V \otimes V$ consists of the identity automorphism and the automorphism $v \rightarrow-v$ for all $v$ in $V$.

Proof. Let $\pi$ be an automorphism of $V$ such that $\pi^{\prime}$ is the identity automorphism of $V \otimes V$. Consider $0 \neq v \in V$, and let $v=v_{1}, v_{2}, \ldots$ be a basis for $V$. With respect to this basis, $\pi$ is represented by a rational matrix $A=\left(a_{i j}\right)$ and $\pi^{\prime}$ is represented by the rational matrix

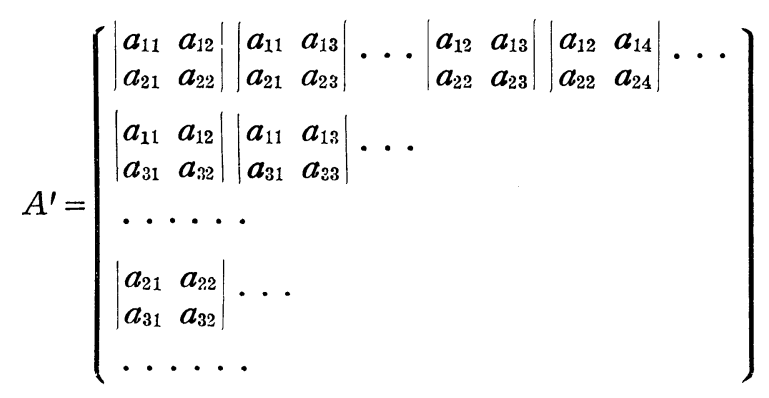

and

$$
v_{1} \pi=a_{11} v_{1}+\cdots+a_{1 n} v_{n} \quad n>3
$$

Consider only the rows and columns of $A^{\prime}$ that are formed by the first $n$ rows and the first $n$ columns of $A$. This matrix is still the identity matrix. Therefore by Lemma $4.1, a_{11}=a_{22}=\cdots=a_{n n}= \pm 1$ and $a_{i j}=0$ for all $i \neq j$ with $i, j<n$. It follows that $v_{1} \pi= \pm v_{1}$. But $v_{1}$ is an arbitrary non-zero element of $V$, and hence $a \pi= \pm a$ for all $a \in V$. Therefore $A$ is a diagonal matrix with the diagonal elemets $d_{i}= \pm 1$. But since $A^{\prime}$ is the identity matrix, either all the $d_{i}=1$ or all the $d_{i}=-1$.

Note that if $n=2$, then

$$
\left[\begin{array}{ll}
a_{11} & a_{12} \\
a_{21} & a_{22}
\end{array}\right] \rightarrow\left|\begin{array}{ll}
a_{11} & a_{12} \\
a_{21} & a_{22}
\end{array}\right|
$$

is the natural homomorphism of the automorphisms of $V$ into the automorphisms of $V \otimes V$. The kernel in this case consists of all the matrices with determinant equal to 1 .

Note also that if $V$ is a quasi-cyclic group, then $V \otimes V=0$. Thus our 
theorem does not hold for arbitrary complete groups.

\section{The Groups $H(\Delta,(\Delta \times \Delta) / K, f)$ and the Class $\mathscr{S}$}

Let $\Delta$ be an abelian group, and let $e$ be the natural (*)-mapping of $\Delta \times \Delta$ into $\Delta \otimes \Delta$, namely $e(\alpha, \beta)=\alpha \otimes \beta$. Let $G=G(\Delta, \Delta \otimes \Delta, e)$, and let $Q=\theta \times(\Delta \otimes \Delta)$. From the propositions in Section 2 it follows that $Z(G) \supseteq Q \supseteq 2 Q \supseteq C(Q)$. Also, by the propositions in Section 4 , if $\Delta$ is torsion free or divisible, then so are $\Delta \times \Delta$ and $G$.

Theorem 5.1. Let $H$ be the group $H(\Delta, N, f)$, where $\Delta$ and $N$ are abelian groups and $f$ is a (*)-mapping of $\Delta \times \Delta$ into $N$ that generates $N$. Let $\psi$ be the unique homomorphism of $\Delta \times \Delta$ onto $N$ such that $f(\alpha, \beta)=e(\alpha, \beta) \psi$ for all $\alpha$ and $\beta$ in $\Delta$. Then the mapping $\sigma$ of $(\alpha, a) \in G(\Delta, \Delta \otimes \Delta, e)$ upon $(\alpha, a \psi)$ is $a$ homomorphism of $G$ onto $H$-the natural homomorphism.

$$
\begin{aligned}
\text { Proof. }[(\alpha, a)+(\beta, b)] \sigma & =(\alpha+\beta, e(\alpha, \beta)+a+b)_{\sigma} \\
& =(\alpha+\beta, e(\alpha, \beta) \psi+a \psi+b \psi) \\
& =(\alpha+\beta, f(\alpha, \beta)+a \psi+b \psi) \\
& =(\alpha, a)_{\sigma}+(\beta, b)_{\sigma} .
\end{aligned}
$$

Let $\mathscr{S}$ be the class of all nilpotent groups of class 2 that have representations $H(\Delta, N, f)$, where $\Delta$ and $N$ are abelian groups and $f$ is a (*)-mapping of $\Delta \times \Delta$ into $N$ that generates $N$. Then by the above theorem, each group in $\mathcal{S}$ is a homomorphic image of a group of the form $G(\Delta, \Delta \otimes \Delta, e)$. In fact, each group in $\mathscr{S}$ has a natural representation of the form

$$
H(\Delta,(\Delta \otimes \Delta) / K, f)
$$

where $K$ is a subgroup of $\Delta \otimes \Delta$ and for all $\alpha$ and $\beta$ in $\Delta$

$$
f(\alpha, \beta)=K+\alpha \otimes \beta .
$$

We shall always choose such a representation, and $H(\Delta,(\Delta \otimes \Delta) / K, f)$ will always denote such a representation.

Let $\mathscr{E}$ be the class of all complete nilpotent groups of class 2 , and let $\mathscr{C}$ and $\mathscr{D}$ be the classed defined in section 3 . Then by Theorem 3.1 and the remarks after the definitions of $\mathscr{C}$ and $\mathscr{D}$,

$$
\mathscr{C} \subseteq \mathscr{D} \subseteq \mathscr{E} \subseteq \mathscr{S}
$$


Now let $G$ be an arbitrary nilpotent group of class 2 . If $g$ is the commutator function, $g(C(G)+a, C(G)+b)=[a, b]$, then $G(G / C, C, g)$ belongs to $\mathcal{S}$. If

$$
\{g \in G: n g \in C(G) \text { for some } n>0\} \subseteq Z(G),
$$

then by Theorem 3.2 in [3] there exists a completion of $G$. Hence $G$ is a subgroup of a group in $\mathscr{S}$.

THEOREM 5.2. Let $\Delta$ and $N$ be abelian groups and let $G$ be a central extension of $N$ by $\Delta$. If $\Delta$ is torsion free or if $N$ contains no elements of order 2 , then $G$ is a subgroup of a group in $\mathcal{S}$.

Proof. $G$ has a representation $G(\Delta, N, f)$, where $f$ is a factor mapping of $\Delta \times \Delta$ into $N$, and $G(\Delta, N, f)$ is a subgroup of $G\left(\Delta, N^{*}, f\right)$, where $N^{*}$ is the abelian completion of $N$. If $\Delta$ is torsion free, then by Lemma 3.4 in [3], $f$ is equivalent to a (*)-mapping $h$ of $\Delta \times \Delta$ into $N^{*}$. If $N$ contains no elements of order 2 , then neither does $N^{*}$, and hence by Theorem 2.1 in [3], $f$ is equivalent to a (*)-mapping $h$ of $\Delta \times \Delta$ into $N^{*}$. Thus in either case

$$
G \subseteq H\left(\Delta, N^{*}, h\right)
$$

where $h$ is a (*)-mapping of $\Delta \times \Delta$ into $N^{*}$. Now $N^{*}$ is a homomorphic image of a rational vector space $D$. Pick a rational vector space $V$ with dimension $d(V \otimes V) \geq d(D)$. Let $\sigma$ be a homomorphism of $V \otimes V$ onto $N^{*}$, and for $\alpha$ and $\beta$ in $V$ let $g(\alpha, \beta)=(\alpha \otimes \beta) \sigma$. Then $g$ is a $(*)$-mapping of $V \times V$ into $N^{*}$ that generates $N^{*}$. Let $J$ be the direct sum of the extensions $H\left(\Delta, N^{*}, h\right)$ and $K(V$, $\left.N^{*}, g\right)$ of $N^{*}$ with amalgamated $N^{*}$. Then $G \subseteq J \in \mathscr{S}$.

If $G=G(\Delta, N, f)$ and the subgroup of $N$ that is generated by $f$ contains no elements of order 2 , then by an entirely similar argument it follows that $G$ is a subgroup of a group in $\mathcal{S}$. If $G$ is a nilpotent group of class 2 and if the commutator subgroup of $G$ contains no elements of order 2 , then by the above theorem $G$ is a subgroup of a group in $\mathscr{S}$.

Suppose that $G$ is a nilpotent group of class 2 that is not a subgroup of a group in $\mathscr{S}$. Then without loss of generality

$$
G=G(\Delta, C, f) \subseteq H\left(\Delta, C^{*}, f\right)
$$

where $C$ is the commutator subgroup of $G, C^{*}$ is the abelian completion of $C$, $C$ contains elements of order $2, \Delta=G / C$ is not torsion free and $f$ is not 
equivalent to a (*)-mapping. Thus $C^{*}=A \oplus B$, where $A$ contains no elements of order 2 and $B$ is a direct sum of quasi-cyclic 2-groups. For $\alpha$ and $\beta$ in $\Delta$ we have

$$
f(\alpha, \beta)=f^{\prime}(\alpha, \beta)+f^{\prime \prime}(\alpha, \beta)
$$

where $f^{\prime}(\alpha, \beta) \in A$ and $f^{\prime \prime}(\alpha, \beta) \in B$. By Theorem 2.1 in [3], $f^{\prime}$ is equivalent to a (*)-mapping $g$ of $\Delta \times \Delta$ into $A$. Hence

$$
f^{\prime}(\alpha, \beta)=g(\alpha, \beta)-t(\alpha+\beta)+t(\alpha)+t(\beta)
$$

where $t$ is a mapping of $\Delta$ into $A$. Thus

$$
f(\alpha, \beta)=g(\alpha, \beta)+f^{\prime \prime}(\alpha, \beta)-t(\alpha, \beta)+t(\alpha)+t(\beta) .
$$

Hence, without loss of generality, $f^{\prime}$ is a (*)-mapping. The trouble is caused by groups of the form $H\left(\Delta, B, f^{\prime \prime}\right)$. For example, it is easy to show that the non-abelian groups of order 8 are not subgroups of groups in $\mathcal{S}$.

For each abelian group $\Delta$, let $\mathscr{S}(\Delta)$ be the class of all groups in $\mathscr{S}$ that have a representation of the form

$$
H(\Delta,(\Delta \otimes \Delta) / K, f) .
$$

If $\Delta_{1}$ and $\Delta_{2}$ are abelian groups, $2 \Delta_{i}=\Delta_{i}$ and $H_{i} \in \mathscr{S}\left(\Delta_{i}\right)$ for $i=1,2$, then without loss of generality

$$
H_{i}=H_{i}\left(\Delta_{i},\left(\Delta_{i} \otimes \Delta_{i}\right) / K_{i}, f_{i}\right) \quad(i=1,2) .
$$

By Proposition 2.6, $C\left(H_{i}\right)=\theta \times\left(\left(\Delta_{i} \times \Delta_{i}\right) / K_{i}\right)$. Thus if $\pi$ is a homomorphism of $H_{1}$ onto $H_{2}$, then $C\left(H_{1}\right)_{\pi}=C\left(H_{2}\right)$ and hence $\pi$ induces a homomorphism of $\Delta_{1}$ onto $\Delta_{2}$. In particular, if $H_{1} \cong H_{2}$, then $\Delta_{1} \cong \Delta_{2}$. Thus if we restrict our attention to the groups $G$ in $\mathscr{S}$ for which $2 G=G$, then if two such groups are isomorphic, they belong to the same $\mathscr{S}(\Delta)$.

THEOREM 5.3. Let $\Delta$ be an abelian group such that $\Delta=2 \Delta$, let $H_{i}=H_{i}(\Delta$, $\left.(\Delta \otimes \Delta) / K_{i}, f_{i}\right)$ for $i=1,2$, and let $\pi$ be a homomorphism of $H_{1}$ onto $H_{2}$. Then for $(\alpha, a)$ in $H_{1}$

$$
(\alpha, a) \pi=\left(\alpha \pi_{1}, \alpha \pi_{2}+a \pi_{3}\right)
$$

where $\pi_{1}$ is an endomorphism of $\Delta$ onto $\Delta, \pi_{3}$ is a homomorphism of $N_{1}=(\Delta \otimes \Delta) /$ $K_{1}$ onto $N_{2}=(\Delta \otimes \Delta) / K_{2}$, and $\pi_{2}$ is a homomorphism of $\Delta$ into $N_{2}$. Moreover, $K_{1} \pi_{1}{ }^{\prime} \subseteq K_{2}$, where $\pi_{1}{ }^{\prime}$ is the endomorphism of $\Delta \otimes \Delta$ that is induced by $\pi_{1}$, and $\pi_{3}$ 
is the homomorphism of $N_{1}$ onto $N_{2}$ that is induced by $\pi_{1}^{\prime} . \pi$ is an isomorphism if and only if $\pi_{1}$ is an automorphism and $K_{1} \pi_{1}{ }^{\prime}=K_{2}$.

Proof. Let $\pi$ be a homomorphism of $H_{1}$ onto $H_{2}$. Then since $C\left(H_{i}\right)=\theta \times$ $\left[(\Delta \otimes \Delta) / K_{i}\right]$ for $i=1,2$ (see Proposition 2.6), it follows that $\left(\theta \times N_{1}\right) \pi=\theta \times N_{2}$ and hence by Theorem 2.1

$$
(\alpha, a) \pi=\left(\alpha \pi_{1}, \alpha \pi_{2}+a \pi_{3}\right)
$$

for all $(\alpha, a)$ in $H_{1}$, where $\pi_{1}$ is an endomorphism of $\Delta$ onto $\Delta, \pi_{3}$ is a homomorphism of $N_{1}$ onto $N_{2}$, and $\pi_{2}$ is a homomorphism of $\Delta$ into $N_{2}$ such that for all $\alpha$ and $\beta$ in $\Delta$

$$
f_{2}\left(\alpha \pi_{1}, \beta \pi_{1}\right)=f_{1}(\alpha, \beta) \pi_{3}
$$

Let $\varphi_{i}$ be the natural homomorphism of $\Delta \otimes \Delta$ onto $N_{i}(i=1,2)$.

$$
\begin{aligned}
(\alpha \otimes \beta) \pi_{1}^{\prime} \varphi_{2} & =K_{2}+(\alpha \otimes \beta) \pi_{1}^{\prime}=K_{2}+\alpha \pi_{1} \otimes \beta \pi_{1} \\
& =f_{2}\left(\alpha \pi_{1}, \beta \pi_{1}\right)=f_{1}(\alpha, \beta) \pi_{3} \\
& =\left(K_{1}+\alpha \otimes \beta\right) \pi_{3}=(\alpha \otimes \beta) \varphi_{1} \pi_{3} .
\end{aligned}
$$

Thus since the $\alpha \otimes \beta$ generate $\Delta \otimes \Delta$, it follows that

$$
\pi_{1}^{\prime} \varphi_{2}=\varphi_{1} \pi_{3}
$$

If $x=y \pi_{1}^{\prime} \in K_{1} \pi_{1}^{\prime}$, then $x \varphi_{2}=y \pi_{1}^{\prime} \varphi_{2}=y \varphi_{1} \pi_{3}=0 \pi_{3}=0$, and hence $x$ belongs to $K_{2}$. Thus

$$
K \pi_{1}^{\prime} \subseteq K_{2}
$$

It follows that $\pi_{3}$ is the unique homomorphism of $N_{1}$ onto $N_{2}$ such that $\pi_{1}^{\prime} \varphi_{2}$ $=\varphi_{1} \pi_{3}$. If $\pi$ is an isomorphism, then by Theorem $2.1, \pi_{1}$ is an automorphism and $\pi_{3}$ is an automorphism. Hence if $x \in K_{2}$, then $x=y \pi_{1}^{\prime}$ for some $y$ in $\Delta \otimes \Delta$, and so $0=x \varphi_{2}=y \pi_{1}^{\prime} \phi_{2}=y \varphi_{1} \pi_{3}$. Thus $0=y \varphi_{1}$, and hence $y \in K_{1}$. Therefore $K_{1} \pi_{1}^{\prime}$ $=K_{2}$. If $\pi_{1}$ is an automorphism and $K_{1} \pi_{1}^{\prime}=K_{2}$, then $\pi_{1}^{\prime}$ is an automorphism and hence $\pi_{3}$ is an isomorphism. Thus by Theorem 2.1, $\pi$ is an automorphism.

Corollary I. If $\Delta=2 \Delta$ and $H_{i}=H_{i}\left(\Delta,(\Delta \times \Delta) / K_{i}, f_{i}\right)$ for $i=1,2$, then $H_{1}$ and $H_{2}$ are isomorphic if and only if there exists an automorphism $\pi$ of $\Delta$ such that $K_{1} \pi^{\prime}=K_{2}$, where $\pi^{\prime}$ is the automorphism of $\Delta \otimes \Delta$ that is induced by $\pi$.

Proof. If $\pi$ is an isomorphism of $H_{1}$ onto $H_{2}$, then by the theorem $K_{1} \pi_{1}{ }^{\prime}=K_{2}$ for an automorphism $\pi_{1}$ of $\Delta$. Conversely suppose that $\pi_{1}$ is an automorphism 
of $\Delta$ for which $K_{1} \pi_{1}^{\prime}=K_{2}$. Let $\pi_{3}$ be the isomorphism of $N_{1}$ onto $N_{2}$ such that $\varphi_{1} \pi_{3}=\pi_{1}^{\prime} \varphi_{2}$. For $(\alpha, a)$ in $H_{1}$ define that

$$
(\alpha, a)_{\pi}=\left(\alpha \pi_{1}, a \pi_{3}\right) .
$$

Then for all $\alpha$ and $\beta$ in $\Delta$

$$
\begin{aligned}
f_{2}\left(\alpha \pi_{1}, \beta \pi_{1}\right) & =K_{2}+\alpha \pi_{1} \otimes \beta \pi_{1}=K_{2}+(\alpha \otimes \beta) \pi_{1}^{\prime} \\
& =(\alpha \otimes \beta) \pi_{1}^{\prime} \varphi_{2}=(\alpha \otimes \beta) \varphi_{1} \pi_{3} \\
& =\left(K_{1}+\alpha \otimes \beta\right) \pi_{3}=f_{1}(\alpha, \beta) \pi_{3} .
\end{aligned}
$$

It follows from Theorem 2.1 that $\pi$ is an isomorphism of $H_{1}$ onto $H_{2}$.

Corollary II. Suppose that $\Delta=2 \Delta$, and let $H=H(\Delta,(\Delta \otimes \Delta) / K, f)$. If $\pi$ is an endomorphism of $H$ onto $H$, then for $(\alpha, a)$ in $H$

$$
(\alpha, a) \pi=(\alpha, a)\left(\begin{array}{cc}
\pi_{1} & \pi_{2} \\
\phi & \pi_{3}
\end{array}\right)=\left(\alpha \pi_{1}, \alpha \pi_{2}+a \pi_{3}\right)
$$

where $\phi$ is the zero homomorphism of $N=(\Delta \otimes \Delta) / K$ into $\Delta, \pi_{2}$ is a homomorphism of $\Delta$ into $N, \pi_{1}$ is an endomorphism of $\Delta$ onto $\Delta$ such that $K \pi_{1}^{\prime} \subseteq K$, and $\pi_{3}$ is the endomorphism $\varphi^{-1} \pi_{1}^{\prime} \varphi$ of $N$ that is induced by $\pi_{1}^{\prime} . \pi$ is an automorphism if and only if $\pi_{1}$ is an automorphism and $K \pi_{1}^{\prime}=K$. In particular, if $K=0$, then $\pi_{3}=\pi_{1}^{\prime}$ and $\pi$ is an automorphism if and only if $\pi_{1}$ is an automorphism.

The corollary is an immediate consequence of the theorem. Let $\pi_{1}$ be an automorphism of $\Delta$ such that $K \pi_{1}^{\prime}=K$, let $\pi_{2}$ be a homomorphism of $\Delta$ into $\Delta \otimes \Delta$, and let $\phi$ be the zero homomorphism of $\Delta \otimes \Delta$ into $\Delta$. Then

$$
\left(\begin{array}{ll}
\pi_{1} & \pi_{2} \\
\phi & \pi_{1}^{\prime}
\end{array}\right)
$$

is an automorphism of $G(\Delta, \Delta \otimes \Delta, e)$ and the mapping

$$
\left(\begin{array}{ll}
\pi_{1} & \pi_{2} \\
\phi & \pi_{1}^{\prime}
\end{array}\right) \longrightarrow\left(\begin{array}{ll}
\pi_{1} & \pi_{2} \varphi \\
\varphi^{-1} \phi & \varphi^{-1} \pi_{1}^{\prime} \varphi
\end{array}\right)
$$

is a homomorphism into the group of all automorphisms of $H(\Delta,(\Delta \otimes \Delta) / K, f)$, where $\varphi$ is the natural homomorphism of $\Delta \otimes \Delta$ onto $(\Delta \otimes \Delta) / K$. This will be a homomorphism onto the group of all automorphisms of $H$ if and only if $\varphi$ induces a homomorphism of $\operatorname{Hom}(\Delta, \Delta \otimes \Delta)$ onto $\operatorname{Hom}(\Delta,(\Delta \otimes \Delta) / K)$.

Let $H=H(\Delta,(\Delta \otimes \Delta) / K, f)$, where $\Delta$ is torsion free. Let $\Delta^{*}$ be the abelian completion of $\Delta$. Since $\Delta^{*}$ is a rational vector space, it follows that $\Delta^{*} \otimes \Delta^{*}$ is 
also a rational vector space. Hence by Proposition 4.7 the natural homomorphism of $\Delta \otimes \Delta$ into $\Delta^{*} \otimes \Delta^{*}$ is an isomorphism. Thus we may consider $\Delta \otimes \Delta$ as a subgroup of $\Delta^{*} \otimes \Delta^{*}$. If $\alpha \otimes \beta \in \Delta^{*} \otimes \Delta^{*}$, then there exists a positive integer $n$ such that $n \alpha, n \beta \in \Delta$ and hence $n^{2}(\alpha \otimes \beta)=n \alpha \otimes n \beta \in \Delta \otimes \Delta$. It follows that $\Delta^{*} \otimes \Delta^{*}$ is the abelian completion of $\Delta \otimes \Delta$. For $\alpha$ and $\beta$ in $\Delta^{*}$ define that $f^{*}(\alpha, \beta)=K+\alpha \otimes \beta$. Then $f^{*}$ is a (*)-mapping of $\Delta^{*} \times \Delta^{*}$ into $B=\left(\Delta^{*} \otimes \Delta^{*}\right) / K$ that generates $B$, and $H$ is a subgroup of $H^{*}\left(\Delta^{*}, B, f^{*}\right)$.

ThEOREM 5.4. If $\Delta$ is a torsion free abelian group and $H=H(\Delta,(\Delta \otimes \Delta) / K$, $f)$, then $H^{*}\left(\Delta^{*},\left(\Delta^{*} \otimes \Delta^{*}\right) / K, f^{*}\right)$ is a completion of $H$. In particular, if $K=0$, then $H^{*}$ is the unique torsion free completion of $H$.

Proof. $H^{*}$ is a nilpotent group of class 2 that contains $H$ and by Proposition $2.1, H^{*}$ is complete. Thus it suffices to show that no proper complete subgroup of $H^{*}$ contains $H$. Let $Q$ be a complete subgroup of $H^{*}$ that contains $H$. If $(\alpha, a) \in Q$ and $n$ is a positive integer, then there exists an element $(\beta, b)$ in $Q$ such that $(\alpha, a)=n(\beta, b)=(n \beta, n b)$. Therefore $\left\{\gamma \in \Delta^{*}:(\gamma, c) \in Q\right\}$ is a complete subgroup of $\Delta^{*}$ that contains $\Delta$, hence it must equal $\Delta^{*}$. Thus, since $\left(\Delta^{*} \otimes \Delta^{*}\right) / K$ is generated by $f^{*}$ it follows that $Q=H^{*}$. If $K=0$, then since $\Delta^{*}$ and, $\Delta^{*} \otimes \Delta^{*}$ are torsion free it follows that $H^{*}$ is torsion free. Thus by a theorem of Vinogradov it follows that $H^{*}$ is the unique torsion free completion of $H$ (see the corollary to Theorem 3.3 in [3]).

The last part of the above theorem may be generalized as follows: Suppose that $H=H(\Delta,(\Delta \otimes \Delta) / K, f)$ and that $\Delta$ and $(\Delta \otimes \Delta) / K$ are torsion free. Then there exists a unique completion $K^{*}$ of $K$ in $\Delta^{*} \otimes \Delta^{*}$, and the mapping of $K+$ $a \in(\Delta \otimes \Delta) / K$ upon $K^{*}+a$ is an isomorphism of $D=(\Delta \otimes \Delta) / K$ into $D^{*}=\left(\Delta^{*} \otimes\right.$ $\left.\Delta^{*}\right) / K$. Thus we may assume that $D$ is a subgroup of $D^{*}$, and that $H$ is a subgroup of $K\left(\Delta^{*}, D^{*}, g\right)$, where $g(\alpha, \beta)=K^{*}+\alpha \otimes \beta$ for all $\alpha$ and $\beta$ in $\Delta^{*}$. It follows that $D^{*}$ is the abelian completion of $D$, and that $K$ is the unique torsion free completion of $H$. This result and the above theorem are good illustrations of what is going on in [3].

\section{The Groups $H(\Delta,(\Delta \otimes \Delta) / K, f)$, Where $\Delta$ is a Rational} Vector Space, and the Class $\mathscr{B}$.

Throughout this section $\Delta$ will always denote a rational vector space with 
dimension $d(\Delta), G$ will denote the group $G(\Delta, \Delta \otimes \Delta, e)$, and $R$ will denote the rational numbers. By the second corollary to Theorem 5.3, the group $a(G)$ of all automorphism of $G$ is isomorphic to the group of all two by two matrices of the form

$$
\left(\begin{array}{ll}
\pi_{1} & \pi_{2} \\
\phi & \pi_{1}^{\prime}
\end{array}\right)
$$

where $\phi$ is the zero homomorphism of $\Delta \otimes \Delta$ into $\Delta, \pi_{1}$ is an automorphism of $\Delta, \pi_{2}$ is a homomorphism of $\Delta$ into $\Delta \otimes \Delta$, and $\pi_{1}^{\prime}$ is the automorphism of $\Delta \otimes \Delta$ that is induced by $\pi_{1}$. Since both $\Delta$ and $\Delta \otimes \Delta$ are rational vector spaces, it follows that $\pi_{1}, \pi_{2}$ and $\pi_{1}^{\prime}$ are linear transformations, and that $\pi_{1}$ and $\pi_{1}^{\prime}$ are nonsingular. Suppose that $d(\Delta)=n$ is finite, then without loss of generality

$$
G=\left\{\left(x_{1}, \ldots, x_{n}, x_{12}, \ldots, x_{1 n}, x_{23}, \ldots, x_{2 n}, \ldots, x_{n-1, n}\right): x_{i}, x_{i j} \in R\right\}
$$

and

$$
\begin{gathered}
\left(x_{1}, \ldots, x_{n}, x_{12}, \ldots, x_{n-1}, n\right)+\left(y_{1}, \ldots, y_{n}, y_{12}, \ldots, y_{n-1, n}\right) \\
=\left(y_{1}+y_{1}, \ldots, x_{n}+y_{n}, x_{1} y_{2}-x_{2} y_{1}+x_{12}+y_{12}, \ldots, x_{n-1} y_{n}\right. \\
\left.-x_{n} y_{n-1}+x_{n-1, n}+y_{n-1}, n\right) .
\end{gathered}
$$

If $\pi$ is an automorphism of $G$, then

$$
\pi=\left(\begin{array}{ll}
\pi_{1} & \pi_{2} \\
\phi & \pi_{1}^{\prime}
\end{array}\right)
$$

where

$$
\begin{aligned}
& \pi_{1}=\left(\begin{array}{cccc}
a_{11} & a_{12} & \cdots & a_{1 n} \\
\cdots & & & \\
a_{n 1} & & & a_{n n}
\end{array}\right) a_{i j} \in R,\left|\pi_{1}\right| \neq 0 \\
& \pi_{1}^{\prime}=\left(\begin{array}{ll}
\left|\begin{array}{ll}
a_{11} & a_{12} \\
a_{21} & a_{22}
\end{array}\right| \cdot \ldots \cdot\left|\begin{array}{ll}
a_{1, n-1} & a_{1, n} \\
a_{2, n-1} & a_{2, n}
\end{array}\right| \\
\ldots & \ldots \\
\mid a_{n-1,1} & a_{n-1,2} \\
a_{n, 1} & a_{n, 2}
\end{array}|\ldots| \begin{array}{ll}
a_{n-1, n-1} & a_{n-1, n} \\
a_{n, n-1} & a_{n, n}
\end{array} \mid\right)
\end{aligned}
$$

$\phi$ is the $\left(n^{2}-n\right) / 2$ by $n$ zero matrix, and $\pi_{2}$ is an $n$ by $\left(n^{2}-n\right) / 2$ rational matrix. Also $|\pi|=\left|\pi_{1}\right|\left|\pi_{1}^{\prime}\right|=\left|\pi_{1}\right|^{n}$ (Proposition 4.10). Thus the group $a(G)$ is a fairly decent subgroup of the full linear group of $\left(n^{2}+n\right) / 2 \times\left(n^{2}+n\right) / 2$ non-singular rational matrices. If $d(\Delta)$ is infinite, then the above has a straightforward generalization. 
Lemma 6.1. For the elements $X$ and $Y$ in $G \backslash C(G)$ the following are equivalent:

(a) $X+Y=Y+X$.

(b) $m X \equiv n Y \bmod C(G)$ for some non-zero integers $m$ and $n$.

Proof. $X=(\alpha, a)$ and $Y=(\beta, b)$, where $\alpha \neq \theta \neq \beta$. Clearly (a) is satisfied if and only if $0=e(\alpha, \beta)=\alpha \otimes \beta$, but this is true if and only if $\alpha$ and $\beta$ are dependent (special case III of Proposition 4.6) and this is equivalent to (b).

Theorem 6.1. $G$ is directly indecomposable and $G$ is not a direct sum of proper central extensions of $C(G)$ with amalgamated $C(G)$.

Proof. Suppose (by way of contradiction) that $G=G_{1} \oplus G_{2}$, where $G_{i} \neq 0$. $Z(G)=Z\left(G_{1}\right) \oplus Z\left(G_{2}\right)$. If $Z\left(G_{1}\right)=G_{1}$, then $G_{1} \subseteq \theta \times(\Delta \otimes \Delta)$. If $(\alpha, a)$ is in $G$, then $(\alpha, a)=(\theta, x)+(\alpha, y)$, where $(\theta, x) \in G_{1}$ and $(\alpha, y) \in G_{2}$. Since $Z(G)=C(G)$ (Corollary II of Lemma 6.3), it follows that $G_{1}$ is generated by elements in $G_{2}$, a contradiction. Thus there exist $a \in G_{1} \backslash Z(G)$ and $b \in G_{2} \backslash Z(G)$, and $a+b=b+a$. Therefore, by Lemma $6.1, m a=n b \bmod Z(G)$ for some non-zero integers $m$ and $n$. Thus $m a=n b+d_{1}+d_{2}$, where $d_{i} \in Z\left(G_{i}\right)$ for $i=1,2$. Since the $Z\left(G_{i}\right)$ are complete, there exists $e_{i} \in Z\left(G_{i}\right)$ such that $m e_{1}=d_{1}$ and $n e_{2}=d_{2}$. Therefore $m\left(a-e_{1}\right)=n\left(b+e_{2}\right)$ belongs to $G_{1} \cap G_{2}$, and hence $a=e_{1} \in Z\left(G_{1}\right)$, a contradiction. Therefore $G$ is directly indecomposable.

Finally suppose that $G$ is a direct sum of proper central extensions $G_{1}$ and $G_{2}$ of $C(G)$ with amalgamated $C(G)$. Pick $X \in G_{1} \backslash C(G)$ and $Y \in G_{2} \backslash C(G)$. Then $X$ and $Y$ satisfy (a) of Lemma 6.1, but not (b), a contradiction.

Recall that $\mathscr{D}$ is the class of all complete nilpotent groups $H$ of class 2 such that $H / C(H)$ is torsion free. It is now clear that $\mathscr{D}$ consists of all those groups in $\mathscr{S}$ that have a representation of the form

$$
H(\Delta,(\Delta \oplus \Delta) / K, f)
$$

where $\Delta$ is a rational vector space and $f$ is the natural (*)-mapping. Such an $H$ is torsion free if and only if $K$ is a subspace of $\Delta \otimes \Delta$. The structure of the group of all automorphisms of $H$ is given by the second Corollary to Theorem 5.3.

LeMMA 6.2. If $\tau$ is a homomorphism of $\Delta$ into $(\Delta \otimes \Delta) / K$, where $K$ is a subspace of $\Delta \otimes \Delta$, then there exists a homomorphism $\sigma$ of $\Delta$ into $\Delta \otimes \Delta$ such that 
$\tau=\sigma \varphi$, where $\varphi$ is the natural homomorphism of $\Delta \otimes \Delta$ onto $(\Delta \otimes \Delta) / K$.

Proof. Let $x_{1}, x_{2}, \ldots$ be a basis for $\Delta$. In each coset $x_{i} \tau$ of $(\Delta \otimes \Delta) / K$ pick a representative $a_{i}, x_{i} \tau=K+a_{i}$. Let $\sigma$ be the homomorphism of $\Delta$ into $\Delta \otimes \Delta$ that is determined by the mapping $x_{i} \rightarrow a_{i}$. Then $x_{i} \sigma \varphi=x_{i} \tau$, and it follows that $\sigma \varphi=\tau$.

Corollary. If $K$ is a subspace of $\Delta \otimes \Delta$, then there is a natural homomorphism of a subgroup of the automorphism group of $G(\Delta, \Delta \otimes \Delta, e)$ onto the group of all automorphisms of $H(\Delta,(\Delta \otimes \Delta) / K, f)$.

This follows at once from the Lemma and the remarks after the second Corollary to Theorem 5.3.

TheOREM 6.2. If $d(\Delta)$ is finite, $K$ is a subspace of $\Delta \otimes \Delta$, and $\pi$ is an endomorphism of $H(\Delta,(\Delta \otimes \Delta) / K, f)$ onto itself, then $\pi$ is an automorphism.

Proof. We make repeated use of the second Corollary to Theorem 5.3. First $\pi$ induces an endomorphism $\pi_{1}$ of $\Delta$ onto $\Delta$, and since $d(\Delta)$ is finite, $\pi_{1}$ must be an automorphism. Thus $\pi_{1}^{\prime}$ is an automorphism of $\Delta \otimes \Delta$ and $K \pi_{1}^{\prime} \subseteq K$. Hence, since $d(K)=d\left(K \pi_{1}^{\prime}\right)$ is finite, $K \pi_{1}^{\prime}=K$ and it follows that $\pi$ is an automorphism of $H$.

For each $\theta \neq \alpha \in \Delta$, let $B_{\alpha}=\{\alpha \otimes \beta: \beta \in \Delta\}$. Then the mapping $\beta \rightarrow \alpha \otimes \beta$ is a homomorphism of $\Delta$ onto $B_{\alpha}$ with kernel $R \alpha$. Thus $B_{\alpha}$ is a subspace of $\Delta \otimes \Delta$, and $d\left(B_{\alpha}\right)=d(\Delta)-1$. Let

$$
H=H(\Delta,(\Delta \otimes \Delta) / K, f) .
$$

By the definition of the class $\mathscr{C}, H \in \mathscr{C}$ if and only if $C(H)=Z(H)$

Lemma 6.3. The following are equivalent:

(a) $H$ belongs to $\mathscr{C}$.

(b) $K+\alpha \otimes \beta=K$ for all $\beta$ in $\Delta$ implies that $\alpha=\theta$.

(c) $B_{\alpha} \subseteq K$ implies that $\alpha=\theta$.

Proof. The proof is straightforward. Note that $K$ in (c) can be replaced by the maximal complete subgroup $\bar{K}$ of $K$, because $B_{\alpha}$ is complete.

Corollary I. If $\bar{K}$ is the maximal complete subgroup of $K$ and if $d(\bar{K})<$ $d(\Delta)-1$, then $H$ belongs to $\mathscr{C}$. 
Proof. If $\theta \neq \alpha \in \Delta$, then $B_{\alpha}$ is a subspace of $\Delta \otimes \Delta$, and $d\left(B_{\alpha}\right)=d(\Delta)-1$. Thus if $d(\bar{K})<d(\Delta)-1$, then $K$ satisfies (c).

Corollary II. If $d(\Delta)>1$, then $G(\Delta, \Delta \otimes \Delta, e)$ belongs to $\mathscr{C}$.

Since $d(\bar{K})=0<d(\Delta)-1$, this is an immediate consequence of Corollary I.

For each cardinal number $\lambda$ let

$$
\mathscr{D}_{\lambda}=\{H \in \mathscr{D}: d(H / C(H))=\lambda\}
$$

and let

$$
G_{\lambda}=G(\Delta, \Delta \otimes \Delta, e)
$$

where $d(\Delta)=\lambda$. If $H_{1}$ and $H_{2}$ belong to $\mathscr{D}$, and if $H_{1} \cong H_{2}$, then there exists a cardinal number $\lambda$ such that $H_{1}$ and $H_{2}$ belong to $\mathscr{D}_{\lambda}$. Let $d(\Delta)=\lambda$. We have a correspondence between the groups in $\mathscr{D}_{\lambda}$ and the subgroups of $\Delta \otimes \Delta$. Moreover, by the first Corollary to Theorem 5.3, if $K_{1}$ and $K_{2}$ are subgroups of $\Delta \otimes \Delta$, then the corresponding groups in $\mathscr{D}_{\lambda}$ are isomorphic if and only if there exists an automorphism $\pi$ of $\Delta$ such that $K_{1} \pi^{\prime}=K_{2}$. The group in $\mathscr{D}_{\lambda}$ that is determined by $K$ is torsion free if and only if $K$ is a subspace of $\Delta \otimes \Delta$, and it belongs to $\mathscr{C}$ if and only if $B_{\alpha} \subseteq \bar{K}$ implies that $\alpha=\theta$ for all $\alpha$ in $\Delta$, where $\bar{K}$ is the maximal divisible subgroup of $K$. Each of the following two theorems gives a characterization of $G_{\lambda}$.

Theorem 6.3. For each $H$ in $\mathscr{D}_{\lambda}$ there exists a homomorphism of $G_{\lambda}$ onto $H$ that induces an isomorphism on $G_{\lambda} / C\left(G_{\lambda}\right)$. Moreover, if $K$ is any other group in $\mathscr{D}_{\lambda}$ that satisfies this condition, then $K \cong G_{\lambda}$. In particular, if $\lambda$ is finite and if $K$ is a group in $\mathscr{D}_{\lambda}$ such that every other group in $\mathscr{D}_{\lambda}$ is a homomorphic image of $K$, then $K \cong G_{\lambda}$.

Proof. If $H \in \mathscr{D}_{\lambda}$, then $H$ has a representation $H(\Delta,(\Delta \otimes \Delta) / K, f)$ and the natural homomorphism of $G_{\lambda}$ onto $\mathrm{H}$ (Theorem 5.1) induces an isomorphism on $G_{\lambda} / C\left(G_{\lambda}\right)$. Conversely, suppose that $K \in \mathscr{D}_{\lambda}$ and for each $H \in \mathscr{D}_{\lambda}$ there exists a homomorphism of $K$ onto $H$ that induces an isomorphism on $K / C(K)$. Let $\pi_{1}$ be a homomorphism of $G_{\lambda}$ onto $K$ that induces an isomorphism on $G_{\lambda} / C\left(G_{\lambda}\right)$, and let $\pi_{2}$ be a homomorphism of $K$ onto $G_{\lambda}$ that induces an isomorphism on $K / C(K)$. Then $\pi_{1} \pi_{2}$ is an endomorphism of $G_{\lambda}$ onto $G_{\curlywedge}$ that induces an isomorphism on $G_{\lambda} / C\left(G_{\lambda}\right)$, and hence by the second Corollary to Theorem 5.3. $\pi_{1} \pi_{2}$ is an automorphism. Therefore $\pi_{1}$ is an isomorphism of $G_{\lambda}$ onto $K$.. 
In general, if $H$ and $K$ belong to $\mathscr{D}_{\lambda}$ and $\pi$ is a homomorphism of $H$ onto $K$, then $C(H) \pi=C(K)$, and hence $\pi$ induces a homomorphism of $H / C(H)$ onto $K / C(K)$. In particular, if $\lambda$ is finite, then $\pi$ must induce an isomorphism of $H / C(H)$ onto $K / C(K)$.

REMARK. If $\lambda$ is infinite, then there exists a $K$ in $\mathscr{D}_{\lambda}$ such that every other group in $\mathscr{D}_{\lambda}$ is a homomorphic image of $K$, but $K \neq G_{\lambda}$. For example, $K=R \oplus G_{\lambda}$ has this property. One can also construct a group in $\mathscr{D}_{\lambda} \cap \mathscr{C}$ with this property.

Theorem 6.4. A group $H$ is isomorphic to $G_{\lambda}$ if and only if $H$ is a complete torsion free nilpotent group of class 2 that contains a well ordered set $S$ of elements $s_{1}, s_{2}, \ldots$ such that

(i) $|S|=\lambda$,

(ii) $\left\{C(H)+s_{i} \mid s_{i} \in S\right\}$ is a basis for $H / C(H)$,

(iii) $\left\{\left[s_{i}, s_{j}\right] \mid s_{i}, s_{j} \in S\right.$ and $\left.i<j\right\}$ is a basis for $C(H)$.

Proof. We first show that $G_{\lambda}=G(\Delta, \Delta \otimes \Delta, e)$ has these proportions. Pick and well order a basis $B=\alpha_{1}, \alpha_{2}, \ldots$ for $\Delta$. Then $|B|=\lambda$ and

$$
\left\{\theta \times(\Delta \otimes \Delta)+\left(\alpha_{i}, 0\right) \mid \alpha_{i} \in B\right\}
$$

is a basis for $G_{\lambda} / C\left(G_{\lambda}\right)$, and since $\left[\left(\alpha_{i}, 0\right),\left(\alpha_{j}, 0\right)\right]=\left(\theta, 2 \alpha_{i} \otimes \alpha_{j}\right)$

$$
\left\{\left[\left(\alpha_{i}, 0\right),\left(\alpha_{j}, 0\right)\right] \mid \alpha_{i}, \alpha_{j} \in B \text { and } i<j\right\}
$$

is a basis for $C\left(G_{\lambda}\right)$.

Conversely suppose that $H$ satisfies the conditions in the Theorem. Then, since $H$ is torsion free and $d(H / C(H))=\lambda, H$ belongs to $\mathscr{D}_{\lambda}$ and has a representation

$$
H(\Delta,(\Delta \otimes \Delta) / K, f)
$$

where $K$ is a subspace of $\Delta \otimes \Delta$ and $f(\alpha, \beta)=K+\alpha \otimes \beta$ for all $\alpha$ and $\beta$ in $\Delta$. Let $s_{i}=\left(\alpha_{i}, a_{i}\right)$ be the given subset of $H$ that satisfies (i), (ii), and (iii). Then clearly the $\alpha_{i}$ form a basis for $\Delta$. If $k \in K$, then in $G_{\lambda}=G(\Delta, \Delta \otimes \Delta, e)$ we have

$$
(\theta, k)=\left(\theta, \sum_{i<j} k_{i j}\left(\alpha_{i} \otimes \alpha_{j}\right)\right)
$$

thus under the natural homomorphism of $G_{\lambda}$ onto $H$ 


$$
(\theta, K)=\left(\theta, K+\sum_{i<j} k_{i j}\left(\alpha_{i} \otimes \alpha_{j}\right)\right)=\sum_{i<j} \frac{k_{i j}}{2}\left[s_{i}, s_{j}\right]
$$

But since $\left\{\left[s_{i}, s_{j}\right] \mid i<j\right\}$ is a basis for $C(H)$, it follows that all of the $k_{i j}$ are zero, and hence $K=0$. Therefore $H \cong G$.

Theorem 6.5. Let $G=G_{n}$, where $n$ is finite. Then $G$ is the unique nilpotent group of class 2 such that $G / C(G) \cong \Delta$ and $C(G) \cong \Delta \otimes \Delta$.

Proof. Suppose that $H$ is a nilpotent group of class 2 and that $H / C(H) \cong 4$ and $C(H) \cong \Delta \otimes \Delta$. Then $H$ has a representation $H(\Delta, \Delta \otimes \Delta, f)$ where $f$ is a (*)-mapping ([3] Theorem 2.1.). It follows that $H$ is complete and hence by Theorem $3.1 f$ generates $\Delta \otimes \Delta$.' Thus there exists a unique homomorphism of $\Delta \otimes \Delta$ onto itself such that $f(\alpha, \beta)=(\alpha \otimes \beta) \varphi$ for all $\alpha, \beta \in \Delta$ (Proposition 4.3). Since $d(\Delta \otimes \Delta)=\left(n^{2}-n\right) / 2$ is finite, $\varphi$ is necessarily an automorphism. It follows that the natural homomorphism of $G_{n}$ onto $H$ (in Theorem 5.1) is an isomorphism.

REMARK. If $d(\Delta)=\lambda$ is infinite, then the above theorem is false. For let $\varphi$ be a homomorphism of $\Delta \otimes \Delta$ onto itself that is not $1-1$. Define $f(\alpha, \beta)=$ $(\alpha \otimes \beta) \varphi$ for all $\alpha, \beta \in \Delta$ and let

$$
H=H(\Delta,(\Delta \otimes \Delta) / K(\varphi), f)
$$

It follows easily that $C(H) \cong \Delta \otimes \Delta$ and $H \mid C(H) \cong \Delta$, but by the first Corollary to Theorem $5.3, H \neq G_{\lambda}$.

A group $\mathscr{A}$ of automorphisms of a group $Q$ is transitive if for each pair of non-zero elements $a$ and $b$ in $Q$ there exists a $\sigma \in a$ such that $a \sigma=b$.

Theorem 6.6. Let $H=H(\Delta,(\Delta \otimes \Delta) / K, f) \in \mathscr{D}$ and let $\mathscr{I}$ be the group of automorphisms of $\Delta \otimes \Delta$ that are induced by the automorphisms of 4 . The following are equivalent:

a) $\mathscr{F}$ is transitive modulo $K$,

b) Every element in $C(H)$ is a commutator.

Proof. Clearly (b) holds if and only if for each $Z$ in $(\Delta \otimes \Delta) / K$ there exist $X$ and $Y$ in $\triangle$ such that $Z=K+X \otimes Y$. Thus using the notation that was developed at the beginning of this section, it follows that (b) is equivalent to $\left(\mathrm{b}^{\prime}\right)$ for each $\left(z_{1}, \ldots\right)$ in $\Delta \otimes \Delta$ there exist $\left(x_{1}, \ldots\right)$ and $\left(y_{1}, \ldots\right)$ in $\Delta$ such. 
that $\left(z_{1}, \ldots\right)-\left(x_{1} y_{2}-x_{2} y_{1}, \ldots\right) \in K$.

On the other hand, (a) is true if and only if for each non-zero $\left(z_{1}, \ldots\right)$ in $\Delta \otimes \Delta$ there exists an automorphism $\pi$ of $\Delta$ such that $(1,0,0, \ldots) \pi^{\prime}-$ $\left(z_{1}, \ldots\right) \in K$. Thus $(\mathrm{a})$ is equivalent to

$\left(\mathrm{a}^{\prime}\right)$ for each $\left(z_{1}, \ldots\right)$ in $\Delta \otimes \Delta$ there exist rational numbers $a_{i j}$ such that

$$
\left(\left|\begin{array}{ll}
a_{11} & a_{12} \\
a_{21} & a_{22}
\end{array}\right|,\left|\begin{array}{ll}
a_{11} & a_{13} \\
a_{21} & a_{23}
\end{array}\right|, \cdots\right)-\left(z_{1}, z_{2}\right) \in K
$$

Clearly $\left(a^{\prime}\right)$ and $\left(b^{\prime}\right)$ are equivalent.

COROLlARY. The group $\mathscr{F}$ of all automorphisms of $\Delta \otimes \Delta$ that are induced by the automorphisms of $\Delta \otimes \Delta$ is transitive if and only if each element in the commutator subgroup of $G(\Delta, \Delta \otimes \Delta, e)$ is a commutator.

In section 7 we show that $\mathscr{F}$ (in the Corollary) is transitive if and only if $d(\Delta)=1,2$, or 3 .

\section{Special Cases and Examples}

Let $\Delta$ be a rational vector space, and let $G=G(\Delta, \Delta \otimes \Delta, e)$. If $d(\Delta)=1$, then $\Delta \otimes \Delta=0$, and hence $G \cong \Delta \cong R$, the rational numbers.

Suppose that $d(\Delta)=2$. Then $d(\Delta \otimes \Delta)=1$, and so without loss of generality $\Delta \otimes \Delta=R$. If $K$ is a proper subgroup of $\Delta \otimes \Delta$, then 0 is the maximal complete subgroup of $K$, and $d(0)=0<1=d(\Delta)-1$. Thus by the first Corollary to Lemma 6.3, $H(\Delta,(\Delta \otimes \Delta) / K, f)$ belongs to $\mathscr{C}$ except when $K=\Delta \otimes \Delta$. The mapping

$$
\left(\begin{array}{ll}
a_{11} & a_{12} \\
a_{21} & a_{22}
\end{array}\right) \rightarrow\left|\begin{array}{ll}
a_{11} & a_{12} \\
a_{21} & a_{22}
\end{array}\right|
$$

is a homomorphism of the group of automorphisms of $\Delta$ onto the group of automorphisms of $\Delta \otimes \Delta$. Thus each automorphism of $\Delta \otimes \Delta$ is induced by an automorphism of $\Delta$. In particular, if $A$ and $B$ are subgroups of $\Delta \otimes \Delta$, then $A \cong B$ if and only if there exists an automorphism $\pi$ of $\Delta$ such that $A \pi^{\prime}=B$. Let $\mathscr{D}_{2}^{\prime}$ be the set of all equivalence classes of isomorphic groups in $\mathscr{D}_{2}^{\circ}$, and $R^{\prime}$ be the set of all equivalence classes of isomorphic subgroups of $R$. It follows from the first Corollary to Theorem 5.3 that there is a $1-1$ correspondence between $\mathscr{D}_{2}^{\prime}$ and $R^{\prime}$. Now by Baer's theory on the subgroups of $R$ 
we have a complete set of invariants for $R^{\prime}$ (see [4] pp. 145-149). Consider $H_{i}\left(\Delta,(\Delta \otimes \Delta) / K_{i}, f_{i}\right)(i=1,2)$ in $\mathscr{D}_{2}$. Suppose that $K_{i}$ is of type $\left(x_{1}, x_{2}, \ldots\right)$ and that $K_{2}$ is of type $\left(y_{1}, y_{2}, \ldots\right) . K_{1} \cong K_{2}$ if and only if $\left(x_{1}, x_{2}, \ldots\right)=\left(y_{1}\right.$, $\left.y_{2}, \ldots\right)$, that is, if and only if $x_{i}=y_{i}$ for all but a finite number of $i$ and if $x_{i} \neq y_{i}$, then both $x_{i}$ and $y_{i}$ are integers. Therefore $H_{1} \cong H_{2}$ if and only if $\left(x_{1}\right.$, $\left.x_{2}, \ldots\right)=\left(y_{1}, y_{2}, \ldots\right)$. On the other hand, $(\Delta \otimes \Delta) / K_{1} \cong(\Delta \otimes \Delta) / K_{2}$ if and only if for all $i$

$$
x_{i}=\infty \text { if and only if } y_{i}=\infty \text {. }
$$

Thus for a fixed homomorphic image $N$ of $R$ there are infinitely many nonisomorphic groups $H(\Delta,(\Delta \otimes \Delta) / K, f)$ such that $(\Delta \otimes \Delta) / K=N$.

Lemma 7.1. Let $d(\Delta)=3$ and let $A$ and $B$ be subspaces of $\Delta \otimes \Delta$. If $d(A)$ $=d(B)$, then there exists an automorphism $\pi$ of 4 such that $A \pi^{\prime}=B$.

Proof. Since $d(\Delta \otimes \Delta)=3, d(A)=0,1,2$ or 3 . If $d(A)=d(B)=0$ or 3 , then clearly the identity automorphism of $\Delta$ will do. If $d(A)=d(B)=2$, then $A=R x \oplus R y$, where $x=\left(x_{1}, x_{2}, x_{3}\right)$ and $y=\left(y_{1}, y_{2}, y_{3}\right)$ are independent. It suffices to show that there is an automorphism $\pi$ of $\Delta$ such that $x \pi^{\prime}=(1,0,0)$ and $y \pi^{\prime}=(0,1,0)$. Note that this also takes care of the case where $d(A)=d(B)=1$, and it shows that the group $\mathscr{F}$ of automorphisms of $\Delta \otimes \Delta$ each of which is induced by an automorphism of $\Delta$ is transitive.

$$
x \pi^{\prime}=x A^{\prime}=\left(x_{1}, x_{2}, x_{3}\right)\left(\begin{array}{lll}
a_{11} & a_{12} & a_{13} \\
a_{21} & a_{22} & a_{23} \\
a_{31} & a_{32} & a_{33}
\end{array}\right)^{\prime}=
$$

$\left(x_{1} A_{33}+x_{2} A_{23}+x_{3} A_{13}, x_{1} A_{32}+x_{2} A_{22}+x_{3} A_{12}, x_{1} A_{31}+x_{2} A_{21}+x_{3} A_{11}\right)$, where the $A_{i j}^{\prime}$ are the minors of the matrix $A$. Let $\left(a_{33}, a_{23}, a_{13}\right)=\left(x_{1},-x_{2}, x_{3}\right)$, and let $\left(a_{32}, a_{22}\right.$, $\left.a_{12}\right)=\left(y_{1},-y_{2}, y_{3}\right)$, and choose $a_{11}, a_{21}$ and $a_{31}$ so that $|A|=1$. Then we have $x A^{\prime}=(1,0,0)$ and $y A^{\prime}=(0,1,0)$.

COROLlaRY. If $d(4)=1,2$ or 3 , then every element in the commutator subgroup of $G(\Delta, \Delta \otimes \Delta, e)$ is a commutator.

This is an immediate consequence of Theorem 6.6 and the fact that the group $\mathscr{F}$ of automorphisms of $\Delta \otimes \Delta$ each of which is indued by an automorphism of $\Delta$ is transitive. 
TheоRем 7.1. For a group $H$ and $n=1,2,3$ the following are equivalent:

a) $H$ is isomorphic to $G_{n}=G(\Delta, \Delta \otimes \Delta, \in)$ where $d(\Delta)=n$;

b) $H$ is a torsion free group in $\mathscr{D}_{n}$ and for $a, b \in H \backslash C(H), a+b=b+a$ if and only if $n a \equiv n b \bmod C(H)$ for some non-zero integers $m$ and $n$.

Proof. By Lemma 6.1, $G_{n}$ satisfies (b) with no restriction on $n$. Conversely suppose that $H$ satisfies (b). Without loss of generality

$$
H=H(\Delta,(\Delta \otimes \Delta) / K, f)
$$

and since $H$ is torsion free, $K$ is a subspace of $\Delta \otimes \Delta$. Clearly if $n=1$ or 2 , then $K=0$. Suppose (by way of contradiction) that $n=3$ and $K \neq 0$. Let $\alpha_{1}$, $\alpha_{2}, \alpha_{3}$ be a basis for 4 . By Lemma 7.1 there exists an automorphism $\pi$ of $\Delta$ such that $K \pi \supseteq R\left(\alpha_{1} \otimes \alpha_{2}\right)$. This means that $\left(\alpha_{1}, 0\right)$ and $\left(\alpha_{2}, 0\right)$ commute and clearly $m \alpha_{1} \neq n \alpha_{1}$ for any pair of non-zero integers, a contradiction.

If $d(\Delta)=4$, then $d(\Delta \otimes \Delta)=6$ and a straightforward computation shows that the group $\mathscr{I}$ of induced automorphisms of $\Delta \otimes \Delta$ is not transitive. Thus $\mathscr{F}$ is transitive if $d(\Delta) \leq 3$ and not transitive if $d(\Delta)>3$. It follows that the converse of the Corollary to Lemma 7.1 is valid, and that Theorem 7.1 is false for $n>3$.

Each torsion free complete nilpotent group $H$ of class 2 for which $d(H \mid C(H))$ is finite determines an ordered pair of integers $(n, m)$, where $m=d(C(H))$ and $n=d(H \mid C(H))$. We say that $H$ is of type $(n, m)$. Clearly the groups of type $\left(n,\left(n^{2}-n\right) / 2\right)$ are unique (to within an isomorphism) because they have a representation of the form $G(\Delta, \Delta \otimes \Delta, e)$ where $d(\Delta)=n$. An immediate consequence of Lemma 7.1 is that the groups of type $(3,1)$ and $(3,2)$ are unique. The group of type $(3,2)$ is determined by the $(*)$-mapping

$$
f\left(\left(x_{1}, x_{2}, x_{3}\right),\left(y_{1}, y_{2}, y_{3}\right)\right)=\left(x_{1} y_{2}-x_{2} y_{1}, x_{1} y_{3}-x_{3} y_{1}\right) .
$$

The group of type $(3,1)$ is a direct sum of $R$ and the group of type $(2,1)$. If $d(\Delta)=4$, then the group $\mathscr{F}$ of induced automorphisms of $\Delta \otimes \Delta$ is not transitive. It follows that there exist non-isomorphic groups of type $(4,5)$.

Groups of type $(n, 1)$. Let $G=G(\Delta, R, f)$ where $\Delta$ is an $n$-dimensional rational vector space and $f$ is a (*)-mapping of $\Delta \times \Delta$ into $R$ that generates $R$. Let $\delta_{1}, \ldots, \delta_{n}$ be a basis for $\Delta$, and consider $\alpha=\alpha_{1} \delta_{1}+\cdots+\alpha_{n} \delta_{n}$ and $\beta=$ $\beta_{1} \delta_{1}+\cdots \beta_{n} \delta_{n}$ in $A$. Then 


$$
f(\alpha, \beta)=\sum_{i, j} \alpha_{i} \beta_{j} f\left(\delta_{i}, \delta_{j}\right)=\sum_{i<j}\left(\alpha_{i} \beta_{j}-\alpha_{j} \beta_{i}\right) f\left(\delta_{i}, \delta_{j}\right)
$$

Let $f\left(\delta_{i}, \delta_{j}\right)=a_{i j}$. Then

$$
f(\alpha, \beta)=\left(\alpha_{1}, \ldots, \alpha_{n}\right)\left(a_{i j}\right)\left(\beta_{1}, \ldots, \beta_{n}\right)^{T} .
$$

Thus $f$ is determined by an $n \times n$ skew-symmetric rational matrix $A=\left(a_{i j}\right)$. Moreover, the mapping $(\alpha, a) \rightarrow\left(\left(\alpha_{1}, \ldots, \alpha_{n}\right), a\right)$ is an isomorphism of $G(\Delta$, $R, f$ ) onto $G\left(R_{n}, R, A\right)$, where $R_{n}=R \times R \times \cdots \times R$ ( $n$ factors). Let $\alpha \rightarrow \alpha \cdot P$ be an automorphism of $R_{n}$ (where $P$ is an $n \times n$ non-singular matrix). The mapping $\sigma$ of $(\alpha, a)$ upon $(\alpha P, a)$ is an isomorphism of $G\left(R_{n}, R, A\right)$ onto $G\left(R_{n}, R, P^{-1} A P^{-T}\right)$ and hence we may as well assume that $A$ has the canonical form

$$
\left(\begin{array}{rrrrrr}
0 & 1 & & & \\
-1 & 0 & \multicolumn{2}{c}{} & & \\
& & 0 & 1 & \\
& & -1 & 0 & \\
& & & & . & .
\end{array}\right)
$$

If $n=2 m$ is even, then $C(G)=Z(G)$ if and only if $A$ is non-singular. Thus there exists a unique group of type $(2 m, 1)$ in $\mathscr{C}$, namely the one determined by the non-singular $2 m \times 2 m$ matrix in the above form.

Let $G$ be a group of type $(n, 1)$ and let $2 m$ be the rank of the canonical matrix $A$ that is determined by $G$. Then clearly $G$ is (isomorphic to) a direct sum of the group of type $(2 m, 1)$ in $\mathscr{C}$ and a rational vector space of dimension $n-2 m$. Thus two groups of type $(n, 1)$ are isomorphic if and only if their canonical matrices have the same rank.

It is easy to see that the group $G$ of type $(2 m, 1)$ in $\mathscr{C}$ is a direct sum of $m$ groups of type $(2,1)$ with amalgamated $R$. The group $\mathscr{A}(G)$ of all automorphisms of $G$ is a homomorphic image of the group of all automorphisms of the group of type $\left(2 m, 2 m^{2}-m\right)$. By using Theorem 2.2 , it is easy to show to show that $\mathscr{A}(G)$ is isomorphic to the group of all $(2 m+1) \times(2 m+1)$ rational matrices of the form

$$
\left[\begin{array}{ll}
a_{11} \cdots a_{1,2 m} & q_{1} \\
\cdots \cdots & \\
a_{2 m, 1} \cdots a_{2 m, 2 m} & q_{2 m} \\
0 \cdot \cdots 0 & d
\end{array}\right]
$$


where

$$
A=\left[\begin{array}{l}
a_{11} \cdots a_{1,2 m} \\
a_{2 m, 1} \cdots a_{2 m, 2 m}
\end{array}\right]
$$

is a non-singular matrix that satisfies

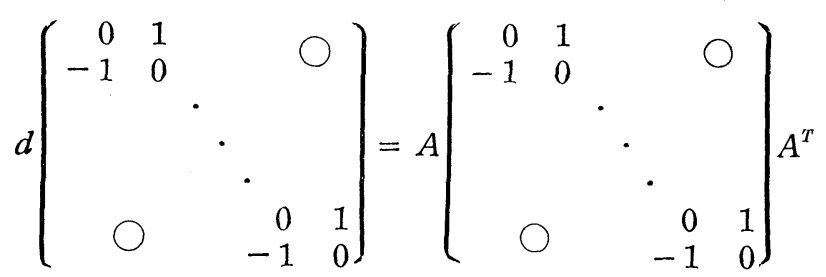

\section{REFERENCES}

[1] R. Baer, Groups with abelian central quotient groups, Trans. Amer. Math. Soc., 44 (1938), pp. 357-386.

[2] R. Baer, Groups with preassigned central and central quotient groups, Trans. Amer. Math. Soc., 44 (1938), pp. 387-412.

[ 3 ] P. Conrad, Completions of groups of class 2, Illinois J. Math. 5 (1961), pp. 212-224.

[4] L. Fuchs, Abelian groups, Budapest (1958). 